\title{
Post-LHC8 supersymmetry benchmark points for ILC physics
}

\author{
Howard Baer ${ }^{1}$ and Jenny List ${ }^{2}$ \\ ${ }^{1}$ University of Oklahoma, Norman, Oklahoma 73019, USA \\ ${ }^{2}$ DESY, Notkestraße 85, 22607 Hamburg, Germany \\ (Received 9 July 2013; published 3 September 2013)
}

\begin{abstract}
We reevaluate prospects for supersymmetry (SUSY) at the proposed International Linear $e^{+} e^{-}$Collider (ILC) in light of the first two years of serious data taking at LHC: LHC7 with $\sim 5 \mathrm{fb}^{-1}$ of $p p$ collisions at $\sqrt{s}=7 \mathrm{TeV}$ and LHC8 with $\sim 20 \mathrm{fb}^{-1}$ at $\sqrt{s}=8 \mathrm{TeV}$. Strong new limits from LHC8 SUSY searches, along with the discovery of a Higgs boson with $m_{h} \simeq 125 \mathrm{GeV}$, suggest a paradigm shift from previously popular models to ones with new and compelling signatures. After a review of the current status of supersymmetry, we present a variety of new ILC benchmark models, including natural SUSY, radiatively driven natural SUSY, NUHM2 with low $m_{A}$, a focus point case from mSUGRA/CMSSM, nonuniversal gaugino mass model, $\tilde{\tau}$ coannihilation, Kallosh-Linde/spread SUSY model, mixed gauge-gravity mediation, normal scalar mass hierarchy, and one example with the recently discovered Higgs boson being the heavy $C P$-even state $H$. While all these models at present elude the latest LHC8 limits, they do offer intriguing case study possibilities for ILC operating at $\sqrt{s} \simeq 0.25-1 \mathrm{TeV}$. The benchmark points also present a view of the widely diverse SUSY phenomena which might still be expected in the post-LHC8 era at both LHC and ILC.
\end{abstract}

DOI: 10.1103/PhysRevD.88.055004

PACS numbers: 12.60.Jv, 14.80.Ly, 11.30.Pb

\section{INTRODUCTION}

\section{A. Motivation}

Supersymmetry (SUSY) is a quantum spacetime symmetry which predicts a correspondence between bosonic and fermionic fields [1-4]. Supersymmetry is particularly appealing for theories of particle physics in that it reduces scalar field quadratic divergences to merely logarithmic. This fact allows for an elegant solution to the notorious gauge hierarchy problem, rendering the weak scale stable against quantum corrections and allowing for stable extrapolations of the standard model (SM) into the far ultraviolet $\left(E \gg M_{\text {weak }}\right.$ ) regime [5,6]. Thus, SUSY provides an avenue for connecting the standard model to ideas of grand unification (GUTs) and/or string theory, and provides a route to unification with gravity via local SUSY, or supergravity theories [7-9].

While models of weak scale supersymmetry are theoretically compelling, we note here that a variety of indirect evidence from experiment has emerged which provides support for the idea of weak scale SUSY:

(i) Gauge coupling unification.-The values of the three SM gauge couplings, measured at energy scale $Q \simeq$ $M_{Z}$ at the CERN LEP collider, when extrapolated to high energy scales via renormalization group (RG) running in the minimal supersymmetric standard model (MSSM) [10], very nearly meet at a point around $Q \simeq 2 \times 10^{16} \mathrm{GeV}$ [11-13]. Unification of gauge couplings is predicted by GUTs and string theories. Gauge coupling unification is violated by numerous standard deviations under SM RG running.

(ii) Precision electroweak measurements:-Fits of precision electroweak observables (EWPO) to SUSY model predictions find accord provided there exists a rather heavy SUSY particle mass spectrum [14]. Meanwhile, models such as minimal technicolor are highly stressed if not ruled out by EWPO.

(iii) Top quark mass and electroweak symmetry breaking:-The electroweak scalar potential is highly constrained in SUSY theories compared to the SM, and it is not immediately clear if electroweak symmetry can be properly broken, yielding the required vector boson and fermion masses while leaving the photon massless. In top-down theories, the soft breaking Higgs mass $m_{H_{u}}^{2}$ is driven to negative values by the large top quark Yukawa coupling, triggering an appropriate breakdown of EW symmetry, provided that the top quark mass $m_{t} \simeq 150-200 \mathrm{GeV}$ [15-18]. The latest measurements find $m_{t}=173.2 \pm 0.9 \mathrm{GeV}$ [19].

(iv) Higgs mass:-Recent data from the CERN LHC [20,21] and Fermilab Tevatron [22] are consistent with discovery of a Higgs boson with $m_{h}=$ $(125.5 \pm 0.5) \mathrm{GeV}$ (combined results), while excluding a SM-like Higgs boson over a vast mass range around this value. In the SM, the Higgs mass is a free parameter, constrained only by unitarity bounds [23]; in SUSY theories, quartic scalar terms are related to gauge couplings so that $m_{h}$ is constrained (within the MSSM, and including radiative corrections) to be $m_{h} \lesssim 135 \mathrm{GeV}$ [24]. The discovery of a light Higgs scalar with mass just below this bound lends credence to the MSSM as a viable effective field theory at the weak scale.

(v) Dark matter:-While none of the SM particles have the right properties to constitute cold dark matter 
(CDM) in the Universe, SUSY theories offer several candidates [25]. These include the neutralino (a WIMP candidate), the gravitino or a singlet sneutrino. In SUSY theories where the strong $C P$ problem is solved via the Peccei-Quinn mechanism, there is the added possibility of mixed (1) axionneutralino [26-28], (2) axion-axino [29-31], or (3) axion-gravitino cold dark matter.

(vi) Baryogenesis:-The measured baryon to photon ratio $\eta \simeq 10^{-10}$ is not possible to explain in the SM. In SUSY theories, three prominent possibilities include (1) electroweak baryogenesis (now nearly excluded by limits on $m_{\tilde{t}_{1}}$ and $m_{h}$ [32]), (2) thermal and nonthermal leptogenesis [33], and (3) Affleck-Dine baryogenesis or leptogenesis $[34,35]$.

\section{B. Some problems for SUSY models}

While the above laundry list is certainly compelling for the existence of weak scale SUSY in nature, we are faced with the fact that at present there is no evidence for direct superparticle production at high energy colliders, especially at the CERN Large Hadron Collider (LHC). The ATLAS and CMS experiments have accumulated $\sim 5 \mathrm{fb}^{-1}$ of integrated luminosity from $p p$ collisions at $\sqrt{s}=7 \mathrm{TeV}$ in 2011 (LHC7), and $\sim 20 \mathrm{fb}^{-1}$ at $\sqrt{s}=8 \mathrm{TeV}$ in 2012 (LHC8). Recent analyses by the CMS experiment [36] using $11.7 \mathrm{fb}^{-1}$ of data at $8 \mathrm{TeV}$ have now excluded $m_{\tilde{g}} \lesssim 1500 \mathrm{GeV}$ in the mSUGRA (also known as CMSSM) model for the case of $m_{\tilde{q}} \simeq m_{\tilde{g}}$, while values of $m_{\tilde{g}} \lesssim 1000 \mathrm{GeV}$ are excluded in the case where $m_{\tilde{q}} \gg m_{\tilde{g}}$. Indeed, as recently as 2010 [37,38], fits of the mSUGRA model to a variety of observables including EWPO, $(g-2)_{\mu}, B$-meson decay branching fractions and neutralino cold dark matter density predicted SUSY to lie exactly in this excluded range. In addition, if the Higgs boson at $\sim 125 \mathrm{GeV}$ turns out to be the light $C P$-even SUSY Higgs, then the minimal versions of gauge-mediated and anomaly mediated SUSY breaking models will likely be ruled out [39], since it is difficult to obtain such large values of $m_{h}$ in these models unless the sparticle mass spectra exist with a lightest MSSM particle of mass greater than about $5 \mathrm{TeV}[40]$.

While the above results may seem disconcerting, at the same time they were not unanticipated by many theorists. Whereas SUSY theories solve a host of problems as mentioned above, they also bring with them considerable phenomenological baggage [41]. Some of these SUSY problems include the following:

(i) The SUSY flavor problem [42].- In SUSY models based on gravity mediation, it is generally expected that large flavor-violating terms will occur in the Lagrangian [43], giving rise to large contributions to the kaon mass difference, and flavor violating decays such as $b \rightarrow s \gamma$ or $\mu \rightarrow e \gamma$. Solutions to the SUSY flavor problem include (1) degeneracy of matter scalar masses, in which case a SUSY
Glashow-Iliopoulos-Maiani mechanism suppresses flavor violation [44], (2) alignment of squark and quark mass matrices [45], or (3) decoupling mainly of first/second generation scalars $\left(m_{\tilde{q}, \tilde{\ell}} \gtrsim\right.$ 5-50 TeV) [46-48]. ${ }^{1}$ Indeed, the SUSY flavor problem provided strong impetus for the development of gauge mediated SUSY breaking (GMSB) and anomaly-mediated SUSY breaking (AMSB) models, where universality of scalars with the same quantum numbers is automatically expected.

(ii) The SUSY CP problem.- In this case, it is expected in gravity mediation that $C P$-violating phases in the soft SUSY breaking terms and perhaps $\mu$ parameter will give rise to large electron and neutron (and other) electric dipole moments. Solutions include dialing the $C P$-violating phases to zero, or decoupling with first generation scalars beyond the few $\mathrm{TeV}$ level. Models such as GMSB and AMSB are again not expected to have complex, $C P$-violating soft terms.

(iii) Proton decay in SUSY GUT theories.-In SUSY GUT theories, the proton is expected to decay to $K^{+} \bar{\nu}$ via colored Higgsino $\tilde{h}$ exchange. The lifetime is expected to occur at levels below experimental limits [49]. Since $\Gamma_{p} \sim m_{p}^{5} / m_{\tilde{h}}^{2} m_{\tilde{q}}^{2}$, large squark masses can again suppress proton decay [50].

(iv) The gravitino problem [51].- In models of gravity mediation, the super-Higgs mechanism generates SUSY breaking by giving the gravitino a mass $m_{3 / 2}$. The gravitino mass sets the scale for the visible sector soft breaking terms, and so one expects sparticle masses of order $m_{3 / 2}$. However, thermal production of gravitinos in the early Universe can lead to either (1) an overproduction of dark matter (here, the gravitinos would decay to the stable lightest SUSY particle (LSP), or even be the LSP), or (2) late-time decays of gravitinos at time scales $\gtrsim 1 \mathrm{~s}$ after the big bang would lead to dissolution of the light nuclei built up during big bang nucleosynthesis (BBN). Solutions to the gravitino problem include (1) a rather low reheat temperature $T_{R} \lesssim$ $10^{5} \mathrm{GeV}$ after inflation so that thermal gravitino production is suppressed [52] (but such low $T_{R}$ values conflict with some baryogenesis mechanisms such as leptogenesis, which seems to require $T_{R} \gtrsim$ $\left.10^{9} \mathrm{GeV}\right),(2)$ a rather light gravitino with $m_{3 / 2} \ll$ $1 \mathrm{GeV}$, which enhances the Goldstino coupling, or (3) a rather heavy gravitino $m_{3 / 2} \gtrsim 5 \mathrm{TeV}$, which lowers the gravitino lifetime so that $\tau_{3 / 2} \lesssim 1 \mathrm{sec}$, and gravitinos decay before BBN [53].

While some proposed solutions solve individual problems listed above (e.g., alignment for the SUSY flavor problem, low $T_{R}$ for the gravitino problem, small phases for the

\footnotetext{
${ }^{1}$ Some degree of alignment or degeneracy would still be needed for the lower portion of this mass range.
} 
SUSY $C P$ problem), there is one solution-decoupling of first/second generation matter scalars-which goes a long way to solving all four. ${ }^{2}$ But what of fine-tuning constraints in SUSY models, which seemingly require sparticle masses near the weak scale $[54,55]$ ?

\section{Fine-tuning in supersymmetric models}

As discussed previously, the most fundamental motivation for weak scale SUSY is that it provides a solution to the gauge hierarchy problem. In SUSY models, the soft SUSY breaking parameters are intimately linked to the breakdown of electroweak symmetry. This fact has motivated hope that the new SUSY matter states should not be too far removed from the weak scale as typified by the $W$, $Z$, and $h$ masses, $\sim 100 \mathrm{GeV}$. However, in the face of increasingly stringent LHC8 mass limits on superpartners, an awkward "little hierarchy" seems to be developing between the weak scale and the superpartner mass scale which has led many to question whether the simple weak scale SUSY picture might be ruled out. This problem is often referred to as the little hierarchy problem (LHP). In this subsection, we will review two approaches to quantify the severeness of the LHP, as manifested by possibly unnatural cancellations in building up the $Z$ - or Higgsboson masses.

\section{High-scale fine-tuning measure $\Delta_{\mathrm{HS}}$ : Minimizing large logs}

In the SM, one may calculate the mass of the Higgs boson as

$$
\left.m_{h}^{2}\right|_{\text {phys }}=\left.m_{h}^{2}\right|_{\text {tree }}+\left.\delta m_{h}^{2}\right|_{\text {rad }}
$$

where $\left.\delta m_{h}^{2}\right|_{\text {rad }}=\frac{c}{16 \pi^{2}} \Lambda^{2}$ and where $\Lambda$ represents the cutoff of quadratically divergent loop diagrams, which provides an upper limit to which the SM is considered a valid effective field theory. The coefficient $c$ depends on the various SM couplings entering particular loop diagrams and here will be taken as $c \simeq 1$. We may define a finetuning measure

$$
\left.\Delta_{\mathrm{SM}} \equiv \delta m_{h}^{2}\right|_{\mathrm{rad}} /\left(m_{h}^{2} / 2\right)
$$

which compares the radiative correction to the physical Higgs boson mass. Requiring $\Delta_{\mathrm{SM}} \lesssim 1$ then requires $\Lambda \simeq 1 \mathrm{TeV}$ : i.e., the $\mathrm{SM}$ should only be valid up to at most the $\mathrm{TeV}$ scale.

Analogous reasoning has been applied to supersymmetric models [56]. In the MSSM,

$$
m_{h}^{2} \simeq \mu^{2}+\left.m_{H_{u}}^{2}\right|_{\text {tree }}+\left.\delta m_{H_{u}}^{2}\right|_{\text {rad }},
$$

\footnotetext{
${ }^{2}$ In gravity mediation, it is expected that the gravitino mass $m_{3 / 2}$ sets the mass scale for the heaviest of the scalars; in this case, multi-TeV scalar masses would proceed from a multi-TeV gravitino mass.
}

where

$\left.\delta m_{H_{u}}^{2}\right|_{\mathrm{rad}} \simeq-\frac{3 f_{t}^{2}}{8 \pi^{2}}\left(m_{Q_{3}}^{2}+m_{U_{3}}^{2}+A_{t}^{2}\right) \ln \left(\Lambda^{2} / M_{\mathrm{SUSY}}^{2}\right)$

and where $\Lambda$ is again the cutoff scale which-inspired by gauge coupling unification-may be taken as high as $M_{\mathrm{GUT}} \simeq 2 \times 10^{16} \mathrm{GeV}$ or even the reduced Planck mass $M_{P}$ and where $M_{\text {SUSY }}^{2} \simeq m_{\tilde{t}_{1}} m_{\tilde{t}_{2}}$. One may again create a fine-tuning measure $\Delta_{\mathrm{KN}} \equiv \delta m_{H_{u}}^{2} /\left(m_{h}^{2} / 2\right)$, following the work of Kitano and Nomura (KN) [57]. Using this, it has been asserted that low electroweak fine-tuning (EWFT) requires rather light third generation squarks:

$\sqrt{m_{\tilde{t}_{1}}^{2}+m_{\tilde{t}_{2}}^{2}} \lesssim 600 \mathrm{GeV} \frac{\sin \beta}{\sqrt{1+R_{t}^{2}}}\left(\frac{\log \frac{\Lambda}{\mathrm{TeV}}}{3}\right)^{-1 / 2}\left(\frac{\Delta_{\mathrm{KN}}}{5}\right)^{1 / 2}$,

where $R_{t}=A_{t} / \sqrt{m_{\tilde{t}_{1}}^{2}+m_{\tilde{t}_{2}}^{2}}$. Taking $\Delta=10$ (i.e., $\Delta^{-1}=$ 0.1 or $10 \%$ EWFT) and $\Lambda$ as low as $20 \mathrm{TeV}$ corresponds to natural SUSY (NS) $[56,58,59]$ :

(i) $m_{\tilde{t}_{i}}, m_{\tilde{b}_{1}} \lesssim 600 \mathrm{GeV}$,

(ii) $m_{\tilde{g}} \lesssim 1.5-2 \mathrm{TeV}$.

The last of these conditions arises because the gluino enters the top-squark radiative corrections $\delta m_{\tilde{t}_{i}}^{2} \sim$ $\left(2 g_{s}^{2} / 3 \pi^{2}\right) m_{\tilde{g}}^{2} \times \log \Lambda$. Setting the $\log$ to unity and requiring $\delta m_{\tilde{t}_{i}}^{2}<m_{\tilde{t}_{i}}^{2}$ then implies $m_{\tilde{g}} \lesssim 3 m_{\tilde{t}_{i}}$, or $m_{\tilde{g}} \lesssim$ $1.5-2 \mathrm{GeV}$ for $\Delta \lesssim 10$. Taking $\Lambda$ as high as $M_{\mathrm{GUT}}$ leads to even tighter constraints: $m_{\tilde{t}_{1,2}}, m_{\tilde{b}_{1}} \lesssim 200 \mathrm{GeV}$ and $m_{\tilde{g}} \lesssim 600 \mathrm{GeV}$, the latter almost certainly in violation of LHC sparticle search constraints. Since (degenerate) first/ second generation squarks and sleptons enter the Higgs potential only at the two loop level, these can be much heavier: beyond LHC reach and also possibly heavy enough to provide a (partial) decoupling solution to the SUSY flavor and $C P$ problems [46]. The NS models in the post-LHC8 period suffer from three phenomenological problems arising from the very light top and bottom squarks: (1) large SUSY contributions to $B F(b \rightarrow s \gamma)$; (2) small radiative corrections to $m_{h}$, thus making it difficult to generate $m_{h} \simeq 125 \mathrm{GeV}$ at least within the MSSM; and (3) there is so far no sign of light stops or sbottoms despite intensive searches at LHC8.

To bring the $\mathrm{KN}$ fine-tuning measure into closer accord with the measure described below, we redefine it in terms of $m_{Z}^{2} / 2$ instead of in terms of $m_{h}^{2} / 2$, so that $\Delta_{\mathrm{HS}} \simeq$ $\delta m_{H_{u}}^{2} /\left(m_{Z}^{2} / 2\right)$, where HS stands for "high scale." The quantity $\Delta_{\mathrm{HS}}$ is a rather severe measure of EWFT in that it does not account for possible correlations amongst HS parameters which may lead to built-in cancellations between $m_{H_{u}}^{2}(\Lambda)$ and $\delta m_{H_{u}}^{2}$. As an example, the boundary condition $m_{H_{u}}^{2}\left(\Lambda=M_{\mathrm{GUT}}\right)=m_{0}$-as in the mSUGRA/ CMSSM model-leads to large cancellations in $m_{H_{u}}^{2}\left(M_{\text {weak }}\right)$ in what has become known as the focus point 
(FP) region [60]. In the FP region, it is argued that the reduced EWFT in the $m_{0}$ direction allows for squark/slepton masses far in excess of the values expected from $\Delta_{\mathrm{HS}}$.

The lesson is that we must remember that HS models such as mSUGRA, NUHM2 etc. are nothing more than effective field theories, albeit ones that are valid up to energy scales $Q \lesssim M_{\mathrm{GUT}}$. In this class of models, the soft parameters merely serve to parametrize our ignorance of their true origin within some more fundamental theory. In the ultimate theory where the soft SUSY breaking parameters (and other parameters) are derived quantities, then correlations between soft parameters may exist which allow for large cancellations between $m_{H_{u}}^{2}(\Lambda)$ and $\delta m_{H_{u}}^{2}$ [61]; in this case, the weak scale value of $m_{H_{u}}^{2}$ becomes more relevant to fine-tuning discussions.

\section{Weak-scale fine-tuning and the little hierarchy problem}

A more conservative EWFT measure has been advocated in Refs. [62-64]. Minimization of the MSSM scalar potential, including radiative corrections, leads to the well-known relation

$$
\frac{m_{Z}^{2}}{2}=\frac{m_{H_{d}}^{2}+\Sigma_{d}^{d}-\left(m_{H_{u}}^{2}+\sum_{u}^{u}\right) \tan ^{2} \beta}{\tan ^{2} \beta-1}-\mu^{2} .
$$

Noting that all entries in Eq. (6) are defined at the weak scale, the electroweak fine-tuning parameter

$$
\Delta_{\mathrm{EW}} \equiv \max _{i}\left|C_{i}\right| /\left(m_{Z}^{2} / 2\right)
$$

may be constructed, where $C_{H_{d}}=m_{H_{d}}^{2} /\left(\tan ^{2} \beta-1\right)$, $C_{H_{u}}=-m_{H_{u}}^{2} \tan ^{2} \beta /\left(\tan ^{2} \beta-1\right)$, and $C_{\mu}=-\mu^{2}$. Also, $C_{\Sigma_{u}^{u}(k)}=-\sum_{u}^{u}(k) \tan ^{2} \beta /\left(\tan ^{2} \beta-1\right) \quad$ and $\quad C_{\Sigma_{d}^{d}(k)}=$ $\sum_{d}^{d}(k) /\left(\tan ^{2} \beta-1\right)$, where $k$ labels the various loop contributions included in Eq. (6). Complete one-loop expressions for the $\Sigma_{u}^{u}$ and $\Sigma_{d}^{d}$ using the ColemanWeinberg effective potential approach are given in the Appendix of Ref. [64].

Thus, $\Delta_{\mathrm{EW}}$ measures the largest weak scale contribution to the $Z$ mass. Model parameter choices which lead to low values of $\Delta_{\mathrm{EW}}$ are those which would naturally generate a value of $m_{Z} \simeq 91.2 \mathrm{GeV}$. If any ranges of model parameters provide low $\Delta_{\mathrm{EW}}$, then one answers the fundamental question of the LHP: how can it be that $m_{Z}$ and $m_{h} \simeq$ $100 \mathrm{GeV}$ while gluino and squark masses lie at the $\mathrm{TeV}$ or beyond energy scale?

In order to achieve low $\Delta_{\mathrm{EW}}$, it is necessary that $\left|m_{H_{u}}^{2}\right|$, $\mu^{2}$ and $\left|\Sigma_{u}^{u}\right|$ all be nearby to $m_{Z}^{2} / 2$ to within a factor of a few $[62,64]$. This implies the following:

(i) $|\mu|$ is favored to be in the $100-300 \mathrm{GeV}$ range (the closer to $m_{Z}$ the better).

(ii) $\left|m_{H_{u}}^{2}\right|_{\text {weak }} \simeq(100-300)^{2} \mathrm{GeV}^{2}$. Such a small value of $m_{H_{u}}\left(M_{\text {weak }}\right)$ only occurs in the FP region of
mSUGRA, i.e., at very large $m_{0}$. In nonuniversal Higgs models (NUHM2), $m_{H_{u}}^{2}$ can be driven radiatively to small negative values at any $m_{0}$ and $m_{1 / 2}$ values.

(iii) To minimize the largest of radiative corrections $\Sigma_{u}^{u}\left(\tilde{t}_{1,2}\right)$, large stop mixing $A_{0} \simeq \pm 1.6 m_{0}$ is required. The large mixing both softens the topsquark radiative corrections while raising $m_{h}$ up to the $\sim 125 \mathrm{GeV}$ level.

The measure $\Delta_{\mathrm{EW}}$ listed above is created from only weak scale MSSM parameters; it contains no information about any possible high scale origin of the soft parameters. In this sense, low $\Delta_{\mathrm{EW}}$ captures a minimal, non-negotiable EWFT required of even high scale SUSY models.

Constrained models such as mSUGRA, mGMSB, and mAMSB have all been found to be highly fine-tuned under $\Delta_{\mathrm{EW}}$. However, models like NUHM2 allow for $\Delta_{\mathrm{EW}}$ as low as 5-10 to be generated. For such cases-under the $\Delta_{\mathrm{EW}}$ measure-the little hierarchy is not a problem ${ }^{3}$ : the necessary condition for low EWFT using $\Delta_{\mathrm{EW}}$ is that only $|\mu|$, $m_{H_{u}}\left(M_{\text {weak }}\right)$ and the various $\Sigma_{u}^{u}$ need be close to the $m_{Z}, m_{h}$ scale.

The low $\Delta_{\mathrm{EW}}$ models are typified by the presence of light Higgsinos $m_{\tilde{\chi}_{1}^{ \pm}}, m_{\tilde{\chi}_{1,2}^{0}} \simeq 100-300 \mathrm{GeV}$. Also, top squark masses can be significantly heavier than in NS models, with $m_{\tilde{t}_{1}} \simeq 1-2 \mathrm{TeV}$ and $m_{\tilde{t}_{2}} \simeq 2-4 \mathrm{TeV}$. Likewise, gluino masses tend to be bounded by about $5 \mathrm{TeV}$ lest they contribute too much to uplifting the top squark masses. The spectrum of Higgsinos is highly compressed amongst themselves: in models with gaugino mass unification, the Higgsino mass gaps are typically $\sim 10-30 \mathrm{GeV}$, since $\left|M_{3}\right| \lesssim 5 \mathrm{TeV}$ requires also upper limits on $M_{1}$ and $M_{2}$. If gaugino mass unification is relaxed, then $M_{1}$ and $M_{2}$ can be heavier, leading to even smaller mass gaps as low as the $\mathrm{GeV}$ range. But even for 10-30 GeV mass splittings, the Higgsino decays give rise to very soft visible energy release which makes their detection at LHC very difficult. Also, since $m_{\tilde{g}} \simeq 1-5 \mathrm{TeV}$, $m_{\tilde{t}_{1}} \simeq 1-2 \mathrm{TeV}$ and $m_{\tilde{t}_{2}} \simeq 2-4 \mathrm{TeV}$, these colored SUSY particles may be too massive to be revealed in LHC SUSY searches. However, the spectrum of light Higgsinos should be easily visible to a linear $e^{+} e^{-}$collider operating with $\sqrt{s} \gtrsim 2|\mu|$.

\section{Remainder of this paper}

The remainder of this paper is geared towards presenting a new set of supersymmetry benchmark models suitable for International Linear $e^{+} e^{-}$Collider (ILC) investigations, while maintaining consistency with the latest indirect and direct constraints on supersymmetric models, especially

\footnotetext{
${ }^{3}$ Low $\Delta_{\mathrm{EW}}$ does not guarantee the absence of electroweak finetuning. It is a necessary, but not sufficient, condition for low finetuning. See, e.g., Ref. [61] for further discussion.
} 
taking into account what has been learned from recent LHC searches. In Sec. II, we briefly summarize current indirect constraints on SUSY models, and also discuss the current status of SUSY dark matter. In Sec. III, we present a summary of the most recent results from LHC searches for SUSY and discovery of the Higgs boson. In Sec. IV, we present a variety of new post-LHC8 benchmark points for ILC studies. These new benchmarks reflect a movement away from previous studies within the mSUGRA/CMSSM model. Some models have been selected due to their theoretical motivation (e.g., natural SUSY and its relatives), while others have been selected for their diversity of phenomenology which may be expected at ILC. In Sec. V, we present a brief summary and outlook for physics prospects at the ILC.

\section{INDIRECT CONSTRAINTS ON SUSY MODELS}

In this section, we review briefly indirect constraints on SUSY models from the measurement of the anomalous magnetic moment of the muon, rare $B$-decay branching fractions along with an updated discussion of the role of dark matter in SUSY models.

\section{A. $(g-2)_{\mu}$ status}

The magnetic moment of the muon $a_{\mu} \equiv \frac{(g-2)_{\mu}}{2}$ was measured by the Muon $g-2$ Collaboration [65] and has been found to give a $3.6 \sigma$ discrepancy with SM calculations based on $e^{+} e^{-}$data [66]: $\Delta a_{\mu}=a_{\mu}^{\text {meas }}-$ $a_{\mu}^{\mathrm{SM}}\left[e^{+} e^{-}\right]=(28.7 \pm 8.0) \times 10^{-10}$. When $\tau$-decay data are used to estimate the hadronic vacuum polarization contribution rather than low energy $e^{+} e^{-}$annihilation data, the discrepancy reduces to $2.4 \sigma$, corresponding to $\Delta a_{\mu}=a_{\mu}^{\text {meas }}-a_{\mu}^{\mathrm{SM}}[\tau]=(19.5 \pm 8.3) \times 10^{-10}$.

The SUSY contribution to the muon magnetic moment is [67] $\Delta a_{\mu}^{\mathrm{SUSY}} \sim \frac{m_{\mu}^{2} \mu M_{i} \tan \beta}{M_{\mathrm{SUSY}}^{4}}$ where $i=1,2$ stands for electroweak gaugino masses and $M_{\mathrm{SUSY}}$ is the characteristic sparticle mass circulating in the muon-muon-photon vertex correction: here, $m_{\tilde{\mu}_{L, R}}, m_{\tilde{\nu}_{\mu}}, m_{\tilde{\chi}_{i}^{+}}$, and $m_{\tilde{\chi}_{j}^{0}}$. Attempts to explain the muon $g-2$ anomaly using supersymmetry usually invoke sparticle mass spectra with relatively light smuons and/or large $\tan \beta$ (see, e.g., Ref. [68]). Some SUSY models where $m_{\tilde{\mu}_{L, R}}$ is correlated with squark masses (such as mSUGRA) are now highly stressed to explain the $(g-2)_{\mu}$ anomaly. In addition, since naturalness favors a low value of $|\mu|$, tension again arises between a large contribution to $\Delta a_{\mu}^{\mathrm{SUSY}}$ and naturalness conditions. These tensions motivate scenarios with nonuniversal scalar masses. Of the benchmark scenarios discussed in the following, some feature light smuons which raise $(g-2)_{\mu}$ to its experimental value, while others are compatible with the standard model prediction.

\section{B. $b \rightarrow s \gamma$}

The combination of several measurements of the $b \rightarrow s \gamma$ branching fraction by the Heavy Flavor Averaging Group (HFAG) [69] finds that $B F(b \rightarrow s \gamma)=(3.55 \pm 0.26) \times$ $10^{-4}$. This is somewhat higher than the SM prediction [70] of $B F^{\mathrm{SM}}(b \rightarrow s \gamma)=(3.15 \pm 0.23) \times 10^{-4}$. SUSY contributions to the $b \rightarrow s \gamma$ decay rate come mainly from chargino-top-squark loops and loops containing charged Higgs bosons, and so are large when these particles are light and when $\tan \beta$ is large [71]. Most SUSY model predictions for $B F(b \rightarrow s \gamma)$ decay assume minimal flavor violation; if this assumption is relaxed, then additional flavor-sector contributions to $B F(b \rightarrow s \gamma)$ can occur.

$$
\text { C. } \boldsymbol{B}_{s} \rightarrow \boldsymbol{\mu}^{+} \boldsymbol{\mu}^{-}
$$

Recently, the LHCb collaboration has discovered an excess over the background for the decay $B_{s} \rightarrow \mu^{+} \mu^{-}$ [72]! They find a branching fraction of $B F\left(B_{s} \rightarrow\right.$ $\left.\mu^{+} \mu^{-}\right)=3.2_{-1.2}^{+1.5} \times 10^{-9}$ in accord with the SM prediction of $(3.2 \pm 0.2) \times 10^{-9}$ [73]. In supersymmetric models, this flavor-changing decay occurs through pseudoscalar Higgs $A$ exchange [74,75], and the contribution to the branching fraction from SUSY is proportional to $\frac{(\tan \beta)^{6}}{m_{A}^{4}}$. Thus, the decay is most constraining at large $\tan \beta \simeq 50$ (as occurs in Yukawa-unified models) and at low $m_{A} \simeq 100-200 \mathrm{GeV}$.

$$
\text { D. } B_{u} \rightarrow \boldsymbol{\tau}^{+} \boldsymbol{\nu}_{\boldsymbol{\tau}}
$$

The branching fraction for $B_{u} \rightarrow \tau^{+} \nu_{\tau}$ decay is calculated [76] in the SM to be $B F\left(B_{u} \rightarrow \tau^{+} \nu_{\tau}\right)=(1.10 \pm$ $0.29) \times 10^{-4}$. This is to be compared to the value from the HFAG [69], which finds a measured value of $B F\left(B_{u} \rightarrow\right.$ $\left.\tau^{+} \nu_{\tau}\right)=(1.67 \pm 0.3) \times 10^{-4}$, somewhat beyond-but not disagreeing with-the SM prediction. The main contribution from SUSY arises due to tree-level charged Higgs exchange, and is large at large $\tan \beta$ and low $m_{H^{+}}$.

\section{E. Dark matter}

During the past several decades, a very compelling and simple scenario has emerged to explain the presence of dark matter in the Universe with an abundance roughly five times that of baryonic matter. The WIMP miracle scenario posits that weakly interacting massive particles would be in thermal equilibrium with the cosmic plasma at very high temperatures $T \gtrsim m_{\text {WIMP }}$. As the Universe expands and cools, the WIMP particles would freeze out of thermal equilibrium, locking in a relic abundance that depends inversely on the thermally averaged WIMP (co)annihilation cross section [77]. The WIMP "miracle" occurs in that a weak strength annihilation cross section gives roughly the measured relic abundance provided the WIMP mass is of the order of the weak scale [78]. The lightest neutralino of SUSY models has been touted as a protypical WIMP candidate [79-81]. 
While the WIMP miracle scenario is both simple and engaging, it is now clear that it suffers from several problems in the case of SUSY theories. The first of these is that in general SUSY theories where the lightest neutralino plays the role of a thermally produced WIMP, the calculated relic abundance $\Omega_{\chi} h^{2}$ is in fact typically $2-4$ orders of magnitude larger than the measured abundance $\Omega_{\mathrm{CDM}}^{\text {meas }} h^{2}=0.115 \pm 0.002[82,83]$ in the case of a binolike neutralino, and 1-2 orders of magnitude lower than measurements in the case of wino- or Higgsino-like neutralinos [84]. In fact, rather strong coannihilation, resonance annihilation or mixed bino-Higgsino or mixed wino-bino annihilation is needed to obtain the measured dark matter abundance. Each of these scenarios typically requires considerable large fine-tuning of parameters to gain the measured dark matter abundance [85]. The case where neutralinos naturally give the measured CDM abundance is when one has a bino-like neutralino annihilating via slepton exchange with slepton masses in the 50-70 GeV range: such mass values were long ago ruled out by slepton searches at LEP2 [86].

The second problem with the SUSY WIMP miracle scenario is that it neglects the gravitino, which is an essential component of theories based on supergravity. Gravitinos can be produced thermally at high rates at high reheat temperatures $T_{R}$ after inflation. If $m_{\tilde{G}}>m_{\mathrm{LSP}}$, then gravitino decays into a stable LSP can overproduce dark matter for $T_{R} \geqslant 10^{10} \mathrm{GeV}$. Even at much lower $T_{R} \simeq 10^{5}-10^{10} \mathrm{GeV}$, thermal production of gravitinos followed by late decays (since gravitino decays are suppressed by the Planck scale) tend to dissociate light nuclei produced in the early Universe, thus destroying the successful picture of big bang nucleosynthesis [53].

The third problem is that the SUSY WIMP scenario neglects at least two very compelling new physics effects that would have a strong influence on dark matter production in the early Universe:

(i) The first of these is that string theory seems to require the presence of at least one light $(\sim 10-100 \mathrm{TeV})$ moduli field [87]. The moduli can be produced at large rates in the early Universe and decay at times $\sim 10^{-1}-10^{5} \mathrm{~s}$ after the big bang. Depending on their branching fractions, they could either feed additional LSPs into the cosmic plasma [88], or decay mainly to SM particles, thus diluting all relics present at the time of decay [89].

(ii) The second neglected effect is the strong $C P$ problem, which is deeply rooted in QCD phenomenology [90]. After more than three decades, the most compelling solution to the strong $C P$ problem is the hypothesis of a Peccei-Quinn axial symmetry whose breaking gives rise to axion particles with mass $\sim 10^{-6}-10^{-9} \mathrm{eV}$ [91]. The axions can be produced nonthermally via coherent oscillations (CO) [92-94], and also would constitute a portion of the dark matter. In SUSY theories, the axions are accompanied by $R$-odd spin- $\frac{1}{2}$ axinos $\tilde{a}$ and $R$-even spin-0 saxions $s$ [95]. Thermal production of axinos and $\mathrm{CO}$ production of saxions can either feed more dark matter particles into the cosmic plasma, or inject additional entropy, thus diluting all relics present at the time of decay. Theoretical predictions for the relic abundance of dark matter in these scenarios are available but very model dependent. In the case of mixed axion-neutralino dark matter, it is usually very difficult to lower a standard overabundance of neutralinos, but it is also very easy to bolster a standard underabundance, e.g., by decay-produced neutralino reannihilation at temperatures below standard freeze-out $[28,96]$. This latter case may lead one to consider SUSY models with a standard underabundance of wino-like or Higgsino-like neutralinos as perhaps the more compelling possibility for CDM. In the case of mixed axion-neutralino CDM, it can be very model dependent whether the axion or the neutralino dominates the DM abundance, and cases where there is a comparable admixture of both are possible.

The upshot for ILC or LHC physics is that one should not take dark matter abundance constraints on SUSY theories too seriously at this point in time.

\section{Status of WIMP dark matter searches}

As of spring 2013, a variety of direct and indirect WIMP dark matter detection searches are ongoing. Several experiments-DAMA/Libra, CoGent, Cresst, and CDMS - claim excess signal rates beyond expected backgrounds. These various excesses can be interpreted in terms of a 5-10 GeV WIMP particle, although the four results seem at first sight inconsistent with each other. It is also possible that muon- or nuclear-decay induced neutron backgrounds-which are very difficult to estimatecontribute to the excesses. Numerous theoretical and experimental analyses are ongoing to sort the situation out. A WIMP particle of several $\mathrm{GeV}$ seems hard to accommodate in SUSY theories (but see, e.g., Ref. [97]).

There also exist excesses of positrons in cosmic rays above expected backgrounds, first observed by the Pamela collaboration [98], and later by the Fermi-LAT [99] and AMS [100] experiments. While this excess could be understood in terms of very massive WIMPs of the order of hundreds of $\mathrm{GeV}$, it is unclear at present whether the positrons arise from exotic astrophysical sources such as pulsars [101,102] or simply from rare misidentification of cosmic protons. A further possible indirect WIMP signal is the $130 \mathrm{GeV}$ gamma ray line seen in some portions of Fermi data $[103,104]$. This could be interpreted as $\chi \chi \rightarrow \gamma \gamma$ which occurs via a box diagram in SUSY. While intriguing, this signal seems incompatible with SUSY in that one would also expect a much larger 
continuum distribution of photons from direct WIMP annihilation, [105] which does not seem to appear.

A variety of other direct WIMP search experiments have probed deeply into WIMP-model parameter space, with no apparent excesses above SM background. At this time, the best limits come from the XENON100 experiment [106], which excludes WIMP-proton scattering cross sections of $\sigma(\chi p) \geq 2 \times 10^{-9} \mathrm{pb}$ at $90 \%$ C.L. for $m_{\mathrm{WIMP}} \simeq$ $100 \mathrm{GeV}$. The XENON100, LUX, and CDMS experiments seem poised to decisively probe the SUSY parameter space associated with well-tempered (mixed bino-Higgsino) dark matter $[107,108]$ (as occurs for instance in focus point SUSY of the mSUGRA model) in the current round of data taking.

\section{Gravitino dark matter}

It is possible in SUSY theories that gravitinos are the lightest SUSY particle, and could fill the role of dark matter. In gravity mediation, the gravitino is expected to have mass of the order of the weak scale. In this case, late decays of thermally produced neutralinos into gravitinos are often in conflict with BBN constraints. If the gravitinos are much lighter, well below the $\mathrm{GeV}$ scale, then their Goldstino coupling is enhanced and BBN constraints can be evaded. This scenario tends to occur for instance in gauge-mediated SUSY theories. The simplest GMSB scenarios now appear in conflict with the LHC discovery of a Higgs boson with $m_{h} \simeq 125 \mathrm{GeV}[39,40]$. We will, however, present an example of a nonminimal GMSB model which is compatible with the Higgs mass measurement.

\section{LHC RESULTS}

In this section, we present a very brief summary of the status of LHC searches for SUSY Higgs bosons and for SUSY particles as of mid-2013.

\section{A. Impact of Higgs searches \\ 1. SM-like Higgs scalar}

After the discovery of a new boson in summer 2012 by the ATLAS [21] and CMS experiments [20], the analysis of the full LHC7 and LHC8 data sets showed that it is indeed a Higgs boson with roughly SM-like signal strengths in various channels $[109,110]$. Its mass has been determined to $125.7 \pm 0.3$ (stat) \pm 0.3 (syst) $\mathrm{GeV}$ by $\mathrm{CMS}$, while ATLAS finds $125.5 \pm 0.2$ (stat) $+0.5-0.6$ (syst) GeV. In addition the Tevatron experiments reported a $3 \sigma$ excess in the search for the SM Higgs boson in the $W / Z+b \bar{b}$ channel [22].

Although the observed Higgs boson looks SM-like, the signal strengths in the various channels have currently still large uncertainties of $30 \%$ to $100 \%$ and therefore easily leave room for deviations of up to $10 \%$ or $20 \%$ as they typically appear in the MSSM.

\section{Nonstandard Higgs bosons}

Searches by ATLAS and CMS for $H, A \rightarrow \tau^{+} \tau^{-}$now exclude a large portion of the $m_{A}$ vs $\tan \beta$ plane [111,112]. In particular, the region around $\tan \beta \simeq 50$, which is favored by Yukawa-unified SUSY GUT theories, now excludes $m_{A}<500 \mathrm{GeV}$. For $\tan \beta=10$, the range $120 \mathrm{GeV}<m_{A}<220 \mathrm{GeV}$ is excluded in the $m_{h}^{\max }$ scenario with $M_{\text {SUSY }}=1 \mathrm{TeV}$. ATLAS and CMS also searched for charged Higgs bosons produced in decays of top quarks. Both experiments exclude charged Higgs masses between 90 and about $150 \mathrm{GeV}$ for $\tan \beta \simeq 20$ (and in the case of ATLAS also for $\tan \beta$ below 4 ) in the $m_{h}^{\max }$ scenario with $M_{\mathrm{SUSY}}=1 \mathrm{TeV}$ [113,114]. For $\tan \beta \simeq 10$, no charged Higgs mass is excluded beyond the LEP limit of $80.0 \mathrm{GeV}$ [115].

\section{Impact of Higgs searches on SUSY models}

A Higgs mass of $m_{h} \simeq 125 \mathrm{GeV}$ lies below the value of $m_{h} \simeq 135 \mathrm{GeV}$ which is allowed by calculations within the MSSM. However, such a large value of $m_{h}$ requires large radiative corrections and large mixing in the top squark sector. In models such as mSUGRA, trilinear soft parameters $A_{0} \simeq \pm 2 m_{0}$ are thus preferred, and values of $A_{0} \simeq 0$ would be ruled out [116,117]. In other constrained models such as the minimal versions of GMSB or AMSB, Higgs masses of $125 \mathrm{GeV}$ require even the lightest of sparticles to be in the multi-TeV range [40], as illustrated in Fig. 1.

In the mSUGRA/CMSSM model, requiring a Higgs mass of about $125 \mathrm{GeV}$ pushes the best fit point in $m_{0}$ and $m_{\frac{1}{2}}$ space into the multi-TeV range [116] and makes global fits of the model to data increasingly difficult $[118,119]$. This has provided motivation for extending the MSSM with gauge singlets $[120,121]$ or vectorlike matter [122] both of which allow for somewhat heavier values of $m_{h}$.

While the experimental uncertainty of the mass has shrunken to about $0.5 \mathrm{GeV}$, a considerable theoretical uncertainty needs to be taken into account when comparing this number to Higgs mass predictions calculated from SUSY parameters. We therefore consider Higgs masses in the range of $122-128 \mathrm{GeV}$ to be in agreement with current observations.

Although the interpretation of the $125 \mathrm{GeV}$ particle as the light, $C P$-even Higgs boson $h$ is the most obvious one, it is not the only possibility. With the current precision on the signal strengths and the current limits on the heavy Higgs bosons and SUSY particles, the heavy $C P$-even Higgs boson, $H$, could be SM-like and the one observed at the LHC [117,123-125].

\section{B. Review of sparticle searches at LHC}

\section{Gluinos and first/second generation squarks}

The ATLAS and CMS collaborations have searched for multijet $+E_{T}^{\text {miss }}$ events arising from gluino and squark pair 

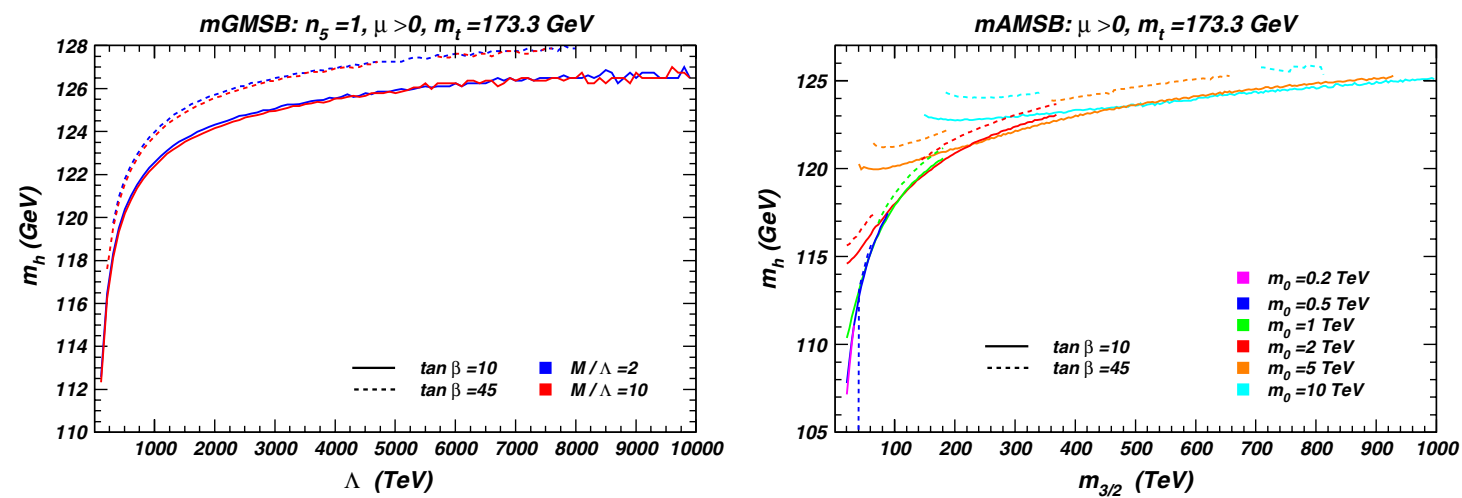

FIG. 1 (color online). Value of $m_{h}$ in mGMSB and in mAMSB versus $\Lambda$ and $m_{3 / 2}$ from [40].

production in $20 \mathrm{fb}^{-1}$ of data taken at $\sqrt{s}=8 \mathrm{TeV}$ $[126,127]$. In a simplified squark-gluino-LSP model, they exclude up to $m_{\tilde{g}} \lesssim 1.4 \mathrm{TeV}$ in the limit of very heavy squark masses, while $m_{\tilde{g}} \lesssim 1.7 \mathrm{TeV}$ is excluded for $m_{\tilde{q}} \simeq m_{\tilde{g}}$. Here, $m_{\tilde{q}}$ refers to a generic first generation squark mass scale, since these are the ones whose production rates depend strongly on valence quark parton distribution functions in the proton.

If the gluino decays dominantly via third generation squarks, the gluino mass limits are somewhat weaker, typically in the range of 1.0 to $1.2 \mathrm{TeV}$, again depending on the exact decay chain [128-133]. Similar limits have been found for the case of intermediate charginos [126].

It has been shown that these limits get considerably weaker if not all squarks are mass degenerate or in case of compressed spectra. In the latter case, the best sensitivity is often obtained from monojet searches [134], and limits on squark masses can reduce to as low as $340 \mathrm{GeV}$.

\section{Sbottom and stop}

Motivated by naturalness, ATLAS and CMS recently put a lot of emphasis on the search for direct production of third generation squarks. They searched for top squarks decaying to $t \tilde{\chi}_{1}^{0}$ [135-137], as well as for $\tilde{t}_{1} \rightarrow b W \tilde{\chi}_{1}^{0}$ [138], $\tilde{t}_{1} \rightarrow b \tilde{\chi}_{1}^{+} \quad[136-139], \quad$ and $\quad \tilde{b}_{1} \rightarrow b Z \tilde{\chi}_{1}^{0} / t W \tilde{\chi}_{1}^{0}$ [140]. In the easiest case, namely for mass differences so large that $m_{\tilde{t}_{1}}>m_{t}+m_{\tilde{\chi}_{1}^{0}}$, stop masses up to about $700 \mathrm{GeV}$ and $\tilde{\chi}_{1}^{0}$ masses up to $200 \mathrm{GeV}$ have been probed. However, the resulting exclusions leave substantial uncovered territory at lower stop masses, where especially the regions near $m_{\tilde{t}_{1}}=m_{t}+m_{\tilde{\chi}_{1}^{0}}$ and $m_{\tilde{t}_{1}}=m_{b}+m_{W}+m_{\tilde{\chi}_{1}^{0}}$ are difficult. The exclusions for $\tilde{b}_{1} \rightarrow b \tilde{\chi}_{1}^{0}$ also apply to top squark pair production in case of $\tilde{t}_{1} \rightarrow b \tilde{\chi}_{1}^{+}$and the $\tilde{\chi}_{1}^{ \pm}$ decays to soft, nearly invisible particles, as would be expected in natural SUSY due to the small mass difference between the Higgsinos. However, these exclusions depend strongly on the assumptions for the chargino and LSP masses. For instance, for $m_{\tilde{\chi}_{1}^{ \pm}}=150 \mathrm{GeV}$ and $m_{\tilde{\chi}_{1}^{0}}$ between about 80 and $140 \mathrm{GeV}$, no stop mass is excluded for the $\tilde{t}_{1} \rightarrow b \tilde{\chi}_{1}^{+}$decay. Stop-LSP mass differences smaller than approximately $40 \mathrm{GeV}$ have not been probed at all so far.

In the context of GMSB with the $\tilde{\chi}_{1}^{0}$ as Higgsino-like next-to-lightest SUSY particle (NLSP) and a gravitino $\tilde{G}$ LSP, ATLAS searched for direct top squark pair production, followed by $\tilde{t}_{1} \rightarrow b \tilde{\chi}_{1}^{+}$or, when kinematically allowed, also $t \tilde{\chi}_{1}^{0}$. Based on $2 \mathrm{fb}^{-1}$, they probe top squark masses up to $600 \mathrm{GeV}$ [141]. This limit relies on the GMSB specific decay of the $\tilde{\chi}_{1}^{0}$ into $Z \tilde{G}$, especially on two (same flavor, opposite sign) leptons consistent with the $Z$ mass.

\section{Electroweakinos}

Direct production of neutralinos, charginos, and sleptons does not rely on colored SUSY partners to be within reach. However, the cross sections at the LHC are significantly lower than for squark and gluino production, so that the obtained limits are considerably weaker. The dominant electroweak production mechanism at the LHC is $\tilde{\chi}_{2}^{0} \tilde{\chi}_{1}^{+}$ production, which has been searched for by ATLAS based on the full $8 \mathrm{TeV}$ data set [142-144], and by CMS based on roughly half the $8 \mathrm{TeV}$ data [145].

The sensitivity of LHC searches for this mode depend strongly on the details of the SUSY spectrum, so that the strongest loophole-free limits on the chargino mass are still the limits of $m_{\tilde{\chi}_{1}^{ \pm}}>103.5 \mathrm{GeV}$ for mass differences larger than $3 \mathrm{GeV}$ and $m_{\tilde{\chi}_{1}^{ \pm}}>92.4 \mathrm{GeV}$ for smaller mass differences down to $60 \mathrm{MeV}$ obtained from LEP data [146]. All LHC searches assume that $\tilde{\chi}_{2}^{0}$ and $\tilde{\chi}_{1}^{ \pm}$are mass degenerate, but have a sizable mass difference of at least 40 to $60 \mathrm{GeV}$ to the $\tilde{\chi}_{1}^{0}$, which is the typical pattern in the case of a binolike LSP. The strongest limits are obtained when assuming that the lighter set of sleptons, including the third generation, is mass degenerate and fulfills $m_{\tilde{l}}=\left(m_{\tilde{\chi}_{1}^{ \pm}}-m_{\tilde{\chi}_{1}^{1}}\right) / 2$, which maximizes the lepton momenta and thus the acceptance. In this case, chargino masses from 100 to $650 \mathrm{GeV}$ are excluded for $m_{\tilde{\chi}_{1}^{0}}=60 \mathrm{GeV}$. For higher LSP masses, the limit does not reach down to the LEP limit. CMS also 
studied the case where the slepton masses are either at 5\% or $95 \%$ of the chargino-LSP mass difference, which leads to a weakening of the limits due to less favorable lepton momentum distributions.

Both collaborations also studied the case of only the $\tilde{\tau}$ (and in case of ATLAS the $\tau$ sneutrino) appearing in the decay chains with a mass halfway between chargino and LSP masses [144,145], and $\tilde{e}$ and $\tilde{\mu}$ being more heavy. In the most optimistic case, i.e., for $m_{\tilde{\tau}}=m_{\tilde{\nu}_{\tau}}=\left(m_{\tilde{\chi}_{1}^{ \pm}}-\right.$ $\left.m_{\tilde{\chi}_{1}^{0}}\right) / 2$, chargino masses up to $350 \mathrm{GeV}$ can be probed for a massless LSP. For $m_{\tilde{\chi}_{1}^{0}}=100 \mathrm{GeV}$, no chargino masses are excluded beyond the LEP limit. This means that in the particularly interesting case of a small $\tilde{\tau}-\tilde{X}_{1}^{0}$ mass difference, as is, e.g., required to obtain a sufficiently low dark matter relic density via $\tilde{\tau}$ coannihilation, the chargino mass is not constrained beyond the LEP results.

Finally, also the possibility that all sleptons are heavier than the chargino has been studied. In this case, chargino and neutralino decay via real or virtual $W$ and $Z$ (or Higgs) bosons, depending on the mass differences. ATLAS [143] and CMS [145] exclude chargino masses up to about 300 to 350 for LSP masses below $70 \mathrm{GeV}$. Above $m_{\tilde{\chi}_{1}^{0}}=120 \mathrm{GeV}$, no chargino masses have been excluded.

\section{Sleptons}

Recently, ATLAS and CMS obtained also first results on slepton pair production [142,145]. For LSP masses below $30 \mathrm{GeV}$, they reach down to the LEP limits and extend up to $300 \mathrm{GeV}$ for left-handed and up to $230 \mathrm{GeV}$ for righthanded selectons and smuons. For higher LSP masses, the LHC exclusions do not connect to the LEP limit, and leave an untouched corridor corresponding to mass differences to the LSP below about $70 \mathrm{GeV}$. It should be noted that this untouched corridor is in the only region where the conditions of this simplified model can be realized without giving up gaugino mass unification at the GUT scale. No slepton masses are excluded for LSP masses above $90 \mathrm{GeV}(150 \mathrm{GeV})$ in the case of righthanded (left-handed) sleptons.

\section{IMPLICATIONS FOR ILC AND BENCHMARK POINTS}

The results from the previous sections, when summarized, yield the following grand picture:

(i) Squarks and gluinos. - Ironically, the strongest LHC limits on sparticle masses apply to the first generation squarks and gluinos, while these are the most remotely connected to the determination of the electroweak scale, and to the weak boson masses. So while $m_{\tilde{g}} \gtrsim 1.5 \mathrm{TeV}$ for $m_{\tilde{q}} \simeq m_{\tilde{g}}$, these limits hardly affect naturalness: e.g., $\Delta_{\mathrm{EW}}<30$ allows for $m_{\tilde{g}}$ as high as $\sim 3-5 \mathrm{TeV}$ and first generation squarks are basically unconstrained so that $m_{\tilde{q}}$ values into the tens of $\mathrm{TeV}$ regime are certainly allowed. (ii) Electroweakinos.-The masses of the electroweakinos-constrained by LEP2 to have $m_{\tilde{\chi}_{1}^{ \pm}}>103.5 \mathrm{GeV}$ - are now also just beginning to be constrained by LHC8 data (subject to certain model assumptions). Some constrained scenarios include (1) models with the gaugino mass unification assumption such that sub-TeV gluinos or first/ second generation squarks would be produced strongly and then cascade decay into electroweakinos; (2) in conjuction with light sleptons with $m_{\tilde{\ell}_{L}}<$ $m_{\tilde{\ell}_{R}}$ where $m_{\tilde{\chi}_{1}^{ \pm}, \tilde{\chi}_{2}^{0}}>m_{\tilde{\ell}}>m_{\tilde{\chi}_{1}^{0}} ;$ and (3) direct $\tilde{\chi}_{1}^{ \pm} \tilde{\chi}_{2}^{0}$ production with decay to trileptons [147] with a nottoo-small $m_{\tilde{\chi}_{1}^{ \pm}, \tilde{\chi}_{2}^{0}}-m_{\tilde{\chi}_{1}^{0}}$ mass gap. In models with light Higgsinos, as motivated by electroweak naturalness, the $m_{\tilde{\chi}_{1}^{0}}, m_{\tilde{\chi}_{2}^{0}}$, and $m_{\tilde{\chi}_{1}^{ \pm}}$can very well be below $300 \mathrm{GeV}$ due to their compressed spectrum: such events are difficult to see at LHC due to prodigious QCD and EW backgrounds. Several of the scenarios proposed below exhibit such a pattern for the light electroweakinos. The heavier electroweakinos, currently unconstrained by LHC8 searches, may be visible at LHC14 in models with light Higgsinos via same-sign diboson production [148]: $\quad p p \rightarrow \tilde{\chi}_{2}^{ \pm} \tilde{\chi}_{4}^{0} \rightarrow\left(W^{ \pm} \tilde{\chi}_{2}^{0}\right)+\left(W^{ \pm} \tilde{\chi}_{1}^{ \pm}\right)+E_{T}^{\text {miss }}$. The proposed benchmarks cover various options in this respect.

(iii) Sleptons. - The most important indication for light sleptons is still $(g-2)_{\mu}$. They are only now beginning to be constrained directly [149] by LHC8 data. If a common matter scalar mass $m_{0}$ at the GUT scale is assumed, then the stringent LHC8 bounds on first and second generation squarks also imply rather heavy sleptons. Most of the scenarios below have heavy sleptons and thus do not explain the $(g-2)_{\mu}$ anomaly. If nonuniversality of matter scalars is assumed, then the slepton masses are completely unconstrained and all sleptons could still lie within reach of the ILC, as illustrated by the $\tilde{\tau}$-coannihilation scenario (STC) and normal scalar mass hierarchy (NMH) benchmarks described below: both these scenarios allow for perfect matches to the observed $(g-2)_{\mu}$ value. In natural SUSY - while the first two slepton generations are expected to be heavy-the $\tilde{\tau}_{1}$ can still be quite light in accord with the required light top and bottom squarks.

(iv) Third generation squarks. - Direct limits on the third generation squarks from LHC8 are becoming increasingly severe and are tightly constraining natural SUSY scenarios (although not radiatively driven natural SUSY). However, if the $\tilde{t}_{1}-\tilde{\chi}_{1}^{0}$ mass gap is small, as expected in stop coannihilation scenarios, then the top squark could very well be in the regime expected from naturalness and be 
accessible to ILC searches. The natural SUSY benchmark and the STC benchmark described in Secs. IVA and IV F give examples with light $\tilde{t}_{1}$ and possibly $\tilde{b}_{1}$ and $\tilde{t}_{2}$.

(v) SUSY Higgses. - The SM-like properties of the newly discovered $125 \mathrm{GeV}$ Higgs scalar suggests that the other SUSY Higgses should be rather heavy. Nevertheless, we present in Sec. IV C a NUHM2 scenario with light $A, H$, and $H^{ \pm}$and in Sec. IV J a case where the heavy $C P$-even Higgs boson has $m_{H} \simeq 125 \mathrm{GeV}$. Also, the STC benchmark features heavy Higgses which should be observable at a $1 \mathrm{TeV} e^{+} e^{-}$collider.

Based on these observations, we propose a set of benchmark points which can be used to illustrate the capabilities of the ILC with respect to supersymmetry, and for future optimization of both machine and detector design. The suggested points all lie outside the limits imposed by LHC8 searches. Some of these scenarios might be discoverable or excluded by upcoming LHC14 searches, while others will be extremely difficult to detect at LHC even with $3 \mathrm{ab}^{-1}$ of data at $\sqrt{s}=14 \mathrm{TeV}$. The spectra for all benchmarks are available online [150] in the SUSY Les Houches Accord format. All figures of the spectra have been obtained with PYSLHA [151].

\section{A. Natural SUSY (NS)}

Natural SUSY (NS) models are characterized by $[58,59,152]:$

(i) a superpotential Higgsino mass parameter $\mu \lesssim$ 100-300 GeV,

(ii) a sub-TeV spectrum of third generation squarks $\tilde{t}_{1}$, $\tilde{t}_{2}$, and $\tilde{b}_{1}$,

(iii) an intermediate scale gluino $m_{\tilde{g}} \lesssim 1.5-3 \mathrm{TeV}$ with $m_{A} \lesssim|\mu| \tan \beta$ and

(iv) multi-TeV first/second generation matter scalars $m_{\tilde{q}, \tilde{\ell}} \simeq 10-50 \mathrm{TeV}$. The last point offers at least a partial decoupling solution to the SUSY flavor and $C P$ problems.

The suggested model parameter space which preserves gauge coupling unification is given by ${ }^{4}$

$$
\begin{array}{ccc}
m_{0}(1,2), & m_{0}(3), & m_{1 / 2}, \\
A_{0}, & \tan \beta, \quad \mu, \quad m_{A} .
\end{array}
$$

Here, we adopt a NS benchmark point as calculated using ISASUGRA 7.83 [153] with parameters $m_{0}(1,2)=$ $13.35 \mathrm{TeV}, m_{0}(3)=0.76 \mathrm{TeV}, m_{1 / 2}=1.38 \mathrm{TeV}, A_{0}=$ $-0.167 \mathrm{TeV}, \quad \tan \beta=23 \mathrm{GeV}, \quad \mu=0.225 \mathrm{TeV}, \quad$ and $m_{A}=1.55 \mathrm{TeV}$. The resulting mass spectrum is listed in

\footnotetext{
${ }^{4}$ The very specific parameter choices used are due to this point being extracted from a scan in Ref. [152]; similar parameter choices will also frequently lead to models with sub-TeV values of $m_{\tilde{t}_{1,2}}$ and $m_{\tilde{b}_{1}}$.
}

Table I and displayed in Fig. 2 for all sparticles (left), and for masses below $500 \mathrm{GeV}$ only (right).

Generic NS models with sub-TeV third generation squarks have difficulty generating (1) $m_{h}=125 \mathrm{GeV}$, (2) $B F(b \rightarrow s \gamma)$, and (3) evading LHC top/bottom squark searches. In this case, some additional contributions to $m_{h}$ or a liberal allowance on theory error would be needed for the Higgs mass along with additional flavor-violating contributions to reconcile $B F(b \rightarrow s \gamma)$ with measurement.

Because of their small mass differences, the Higgsinolike light electroweakinos will tend to look like missing transverse energy to the LHC. The next heavier particle is the $\tilde{t}_{1}$. Since the mass difference $m_{\tilde{t}_{1}}-m_{\tilde{\chi}_{1}^{0}}$ is less than the top mass, the decay $\tilde{t}_{1} \rightarrow b \tilde{\chi}_{1}^{ \pm}$dominates, thus making the signature for $\tilde{t}_{1}$ pair production two acollinear $b$ jets plus missing transverse energy. The relatively compressed mass difference $m_{\tilde{t}_{1}}-m_{\tilde{\chi}_{1}^{ \pm}}=51 \mathrm{GeV}$ allows for evasion of LHC8 top squark search limits.

For ILC, the spectrum of Higgsino-like $\tilde{\chi}_{1}^{ \pm}, \tilde{\chi}_{1}^{0}$, and $\tilde{\chi}_{2}^{0}$ will be accessible for $\sqrt{s} \gtrsim 400-600 \mathrm{GeV}$ via $\tilde{\chi}_{1}^{+} \tilde{\chi}_{1}^{-}$pair production and $\tilde{\chi}_{1}^{0} \tilde{\chi}_{2}^{0}$ mixed production, albeit with a mass gap $m_{\tilde{\chi}_{1}^{ \pm}}-m_{\tilde{\chi}_{1}^{0}} \simeq m_{\tilde{\chi}_{2}^{0}}-m_{\tilde{\chi}_{1}^{0}} \simeq 9 \mathrm{GeV}$ : thus, visible energy released from decays will be small. Specialized cuts allowing for ILC detection of light Higgsinos with small mass gaps have been advocated in Refs. [154,155]; there it is also demonstrated that ILC will be able to measure the values of $\mu$ and $M_{2}$ and show that $|\mu|<M_{2}$.

In the case of very small mass gaps, a hard initial state radiation photon radiated from the initial state may help to lift the signal out of the substantial background of photonphoton induced processes. The experimental performance of this initial state radiation recoil method has been evaluated recently in full simulation of the International Large Detector (ILD) detector in the context of radiative WIMP/ neutralino production $[156,157]$. The cross sections are typically in the few tens of fb region [158] and thus should be detectable in the clean ILC environment. Similar signatures have also been investigated in the context of AMSB for the TESLA technical design report [159].

As $\sqrt{s}$ is increased past $600-800 \mathrm{GeV}$, then also $\tilde{t}_{1} \overline{\tilde{t}}_{1}$, $\tilde{\nu}_{\tau} \overline{\tilde{\nu}}_{\tau}$, and $\tilde{\tau}_{1} \overline{\tilde{\tau}}_{1}$ become successively accessible. This benchmark model can be converted to a model line by varying the GUT-scale third generation mass parameter $m_{0}(3)$ or by varying $\mu$. The light Higgs mass $m_{h}$ can be pushed as high as $\sim 124 \mathrm{GeV}$ if larger values of $m_{0}(3)$ and $\left|A_{0}\right|$ are selected [152].

\section{B. Radiatively driven natural SUSY (RNS)}

Models of RNS are motivated by trying to minimize $\Delta_{\text {EW }}$ while maintaining gauge coupling unification and radiative electroweak symmetry breaking due to the large top squark mass. Low $\Delta_{\mathrm{EW}}$ is obtained by (1) requiring $\mu \simeq 100-300 \mathrm{GeV}$ (lower is more natural), (2) $m_{H_{u}}^{2}$ should run to just small negative values at the weak scale, 
TABLE I. Input parameters and mass spectrum and rates for post-LHC8 benchmark points 1-5. All masses and dimensionful parameters are in $\mathrm{TeV}$ units. All values have been obtained with ISASUGRA.

\begin{tabular}{|c|c|c|c|c|c|}
\hline PMQ & NS & RNS & NUHM2 & mSUGRA & NUGM \\
\hline$m_{0}(1,2), m_{0}(3)$ & $13.35,0.76$ & 5.0 & 10.0 & 10.0 & 3.0 \\
\hline$m_{1 / 2} / M_{1}, M_{2}, M_{3}$ & 1.38 & 0.7 & 0.5 & 0.8 & $0.3,0.25,0.75$ \\
\hline$A_{0}$ & -0.167 & -8.3 & -16.0 & -5.450 & -6.0 \\
\hline $\tan \beta$ & 23 & 10 & 7 & 15 & 25 \\
\hline$\mu$ & 0.225 & 0.11 & 6.0 & 0.234 & 2.36 \\
\hline$m_{A}$ & 1.55 & 1.0 & 0.275 & 9.62 & 3.27 \\
\hline$m_{h}$ & 0.121 & 0.125 & 0.123 & 0.124 & 0.125 \\
\hline$m_{H}$ & 1.56 & 1.0 & 0.278 & 9.69 & 3.29 \\
\hline$m_{H^{ \pm}}$ & 1.55 & 1.0 & 0.286 & 9.69 & 3.29 \\
\hline$m_{\tilde{g}}$ & 3.27 & 1.79 & 1.46 & 2.13 & 1.835 \\
\hline$m_{\tilde{\chi}_{1,2}^{ \pm}}^{0}$ & $0.233,1.18$ & $0.113,0.610$ & $0.474,5.9$ & $0.248,0.70$ & $0.216,2.36$ \\
\hline$m_{\tilde{\chi}_{1,2}^{0}}$ & $0.224,0.232$ & $0.101,0.118$ & $0.237,0.471$ & $0.229,0.247$ & $0.131,0.215$ \\
\hline$m_{\tilde{\chi}_{3,4}^{0,2}}^{10}$ & $0.616,1.18$ & $0.319,0.620$ & $5.9,5.9$ & $0.371,0.72$ & $2.36,2.36$ \\
\hline$m_{\tilde{u}_{L, R}}^{\lambda}$ & $13.58,13.59$ & $5.1,5.3$ & $9.9,10.2$ & $10.0,10.1$ & $3.30,3.31$ \\
\hline$m_{\tilde{t}_{1,2}}$ & $0.284,0.914$ & $1.22,3.55$ & $4.53,7.57$ & $5.80,8.17$ & $1.11,2.29$ \\
\hline$m_{\tilde{d}_{L R}}$ & 13.6, 13.6 & $5.1,5.1$ & $9.9,9.9$ & $10.0,10.1$ & $3.30,3.31$ \\
\hline$m_{\tilde{b}_{1,2}}$ & $0.793,1.25$ & $3.6,5.0$ & $7.58,9.85$ & $8.15,9.88$ & $2.30,2.99$ \\
\hline$m_{\tilde{e}_{L, R}}^{b_{1,2}}$ & $13.4,13.3$ & $5.1,4.8$ & $10.1,9.67$ & $9.99,9.99$ & $3.0,3.0$ \\
\hline$m_{\tilde{\tau}_{1,2}}$ & $0.43,0.53$ & $4.7,5.1$ & $9.61,10.1$ & $9.78,9.89$ & $2.60,2.81$ \\
\hline$\Omega_{\tilde{\tau}_{0}^{0}}^{\text {std }} h^{2}$ & 0.007 & 0.008 & 39 & 0.02 & 1085 \\
\hline$\left\langle\begin{array}{r}\boldsymbol{\sigma} v \\
v\end{array}\right\rangle \times 10^{25}\left[\mathrm{~cm}^{3} / \mathrm{s}\right]$ & 5.5 & 2.3 & 0.0005 & 1.5 & $2.0 \times 10^{-7}$ \\
\hline$\sigma^{\mathrm{SI}}\left(\tilde{\chi}^{0} p\right) \times 10^{9}[\mathrm{pb}]$ & 2.4 & 8.4 & 0.005 & 10.0 & 0.0004 \\
\hline$a_{\mu}^{\mathrm{SUSY}} \times 10^{10}$ & 0.04 & 0.07 & 0.008 & 0.04 & 0.45 \\
\hline$B F(b \rightarrow s \gamma) \times 10^{4}$ & 1.8 & 3.3 & 4.6 & 3.05 & 2.95 \\
\hline$B F\left(B_{S} \rightarrow \mu \bar{\mu}\right) \times 10^{9}$ & 4.3 & 3.8 & 4.0 & 3.8 & 3.9 \\
\hline$B F\left(B_{u} \rightarrow \tau \nu_{\tau}\right) \times 10^{4}$ & 1.3 & 1.3 & 1.2 & 1.3 & 1.3 \\
\hline$\Delta_{\mathrm{EW}}$ & 23 & 16 & 8782 & 321 & 1360 \\
\hline$\Delta_{\mathrm{HS}}$ & 657 & 9810 & 31053 & $2.4 \times 10^{4}$ & 3529 \\
\hline
\end{tabular}

and (3) large mixing in the top squark sector. The large mixing suppresses the radiative corrections $\Sigma_{u}^{u}\left(\tilde{t}_{1}\right)$ and $\sum_{u}^{u}\left(\tilde{t}_{2}\right)$ while increasing $m_{h}$ to $125 \mathrm{GeV}[62,64]$. Thus, these models are typified by the requirement that $\mu$ alone be small $[158,160]$ since the third generation squarks can be far heavier than generic NS models with $m_{\tilde{t}_{1,2}} \simeq$ 1-4 TeV. The RNS model can be realized within the structure of the 2-parameter nonuniversal Higgs mass (NUHM2) model:

$$
m_{0}, \quad m_{1 / 2}, \quad A_{0}, \quad \tan \beta, \quad \mu, \quad m_{A} .
$$

Here, we adopt a benchmark point with parameters $m_{0}=$ $5 \mathrm{TeV}, \quad m_{1 / 2}=0.7 \mathrm{TeV}, A_{0}=-8.3 \mathrm{TeV}, \quad \tan \beta=10$ with $\mu=0.11 \mathrm{TeV}$ and $m_{A}=1 \mathrm{TeV}$. The spectrum is
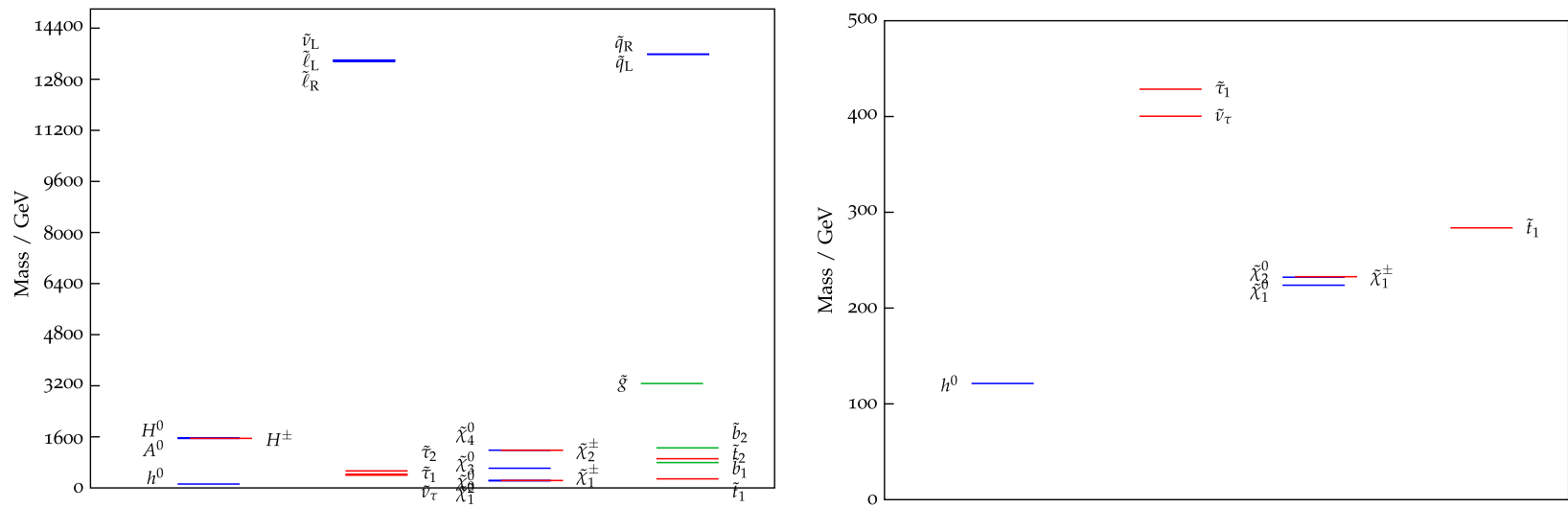

FIG. 2 (color online). Left: Full spectrum of the natural SUSY benchmark. Right: Zoom into the spectrum below 500 GeV. 

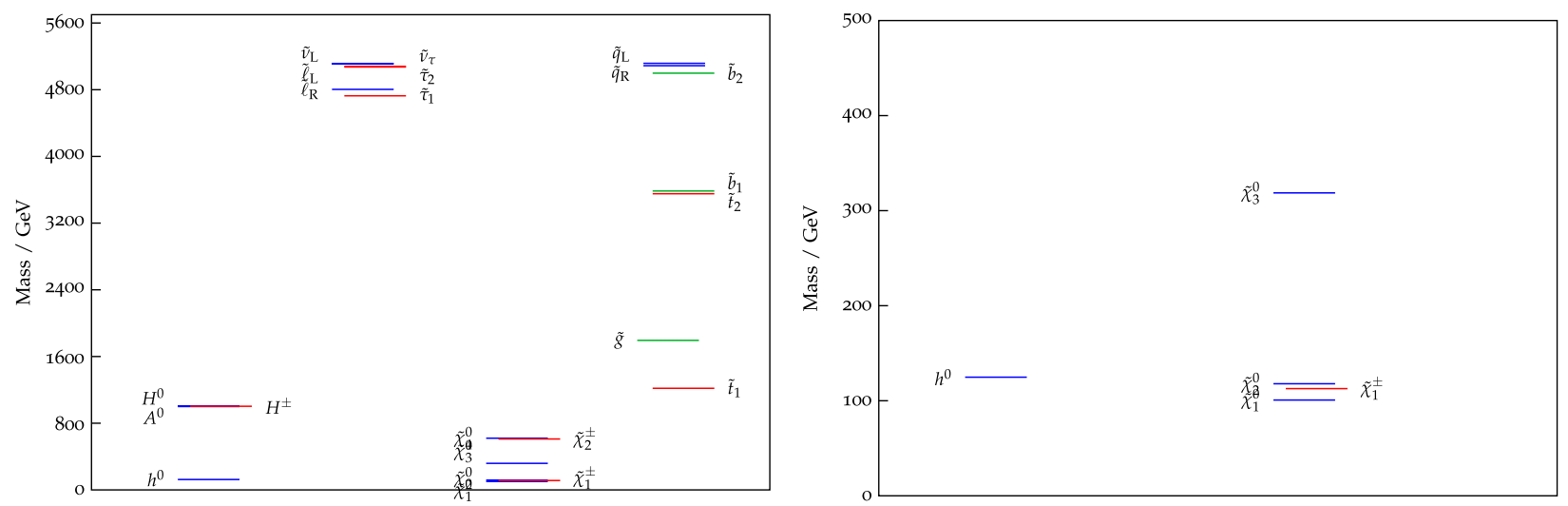

FIG. 3 (color online). Left: Full spectrum of the RNS benchmark. Right: Zoom into the spectrum below $500 \mathrm{GeV}$.

given in Table I. With $\Delta_{\mathrm{EW}}=16$, we have $6.2 \% \mathrm{EW}$ finetuning in $m_{Z}$. The resulting mass spectrum is listed in Table I and displayed in Fig. 3 for all sparticles (left), and for masses below $500 \mathrm{GeV}$ only (right).

RNS models are very difficult to detect at LHC. In contrast to natural SUSY, the third generation scalars are also beyond $1 \mathrm{TeV}$. While the Higgsino-like light charginos and neutralinos are produced at large rates, the very low energy release from their decays will be hard to detect above background levels, making them all look like missing transverse energy. If $m_{\tilde{g}} \lesssim 1.8 \mathrm{TeV}$, then gluino cascade decays should be visible at LHC; however, for RNS models, $m_{\tilde{g}}$ can range as high as $5 \mathrm{TeV}$ while maintaining $\Delta_{\mathrm{EW}} \lesssim 30$ [64]. A unique signature for RNS at LHC14 is same-sign diboson production arising from wino pair production: $p p \rightarrow \tilde{\chi}_{2}^{ \pm} \tilde{\chi}_{4}^{0} \rightarrow\left(W^{ \pm} \tilde{\chi}_{2}^{0}\right)+\left(W^{ \pm} \tilde{\chi}_{1}^{\mp}\right)[148]$.

The ILC turning on at energy $\sqrt{s} \gtrsim 250 \mathrm{GeV}$ should already be able to detect and distinguish $\tilde{\chi}_{1}^{+} \tilde{\chi}_{1}^{-}$and $\tilde{\chi}_{1}^{0} \tilde{\chi}_{2}^{0}$ production as in the NS benchmark model. The small mass gap, angular distribution, and polarization dependence of the signal cross sections may all be used to help establish the Higgsino-like nature of the light $\tilde{\chi}_{1}^{ \pm}, \tilde{\chi}_{2}^{0}$, and $\tilde{\chi}_{1}^{0}$. In addition, the $\tilde{\chi}_{3}^{0}$ is accessible in mixed production with the lighter neutralinos already at $\sqrt{s} \gtrsim 850 \mathrm{GeV}$.

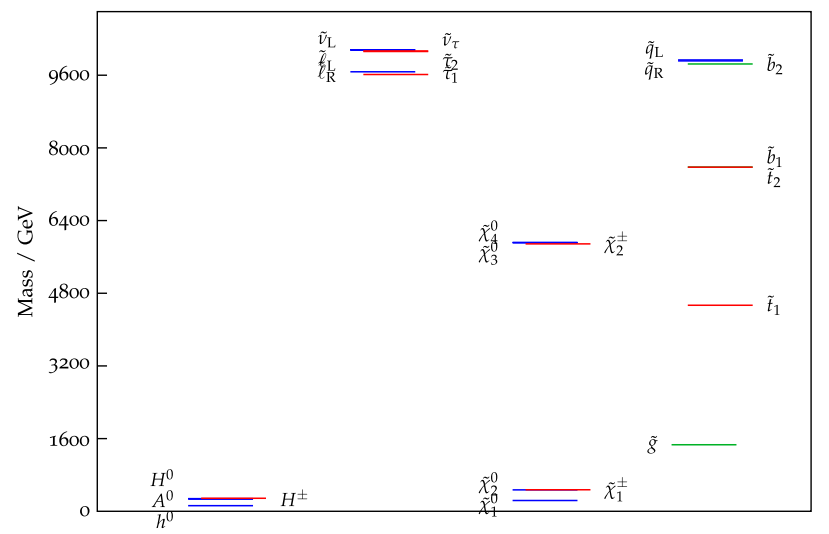

Phenomenologically similar scenarios-which are even more minimal in the sense that the $\tilde{\chi}_{3}^{0}$ and the $\tilde{t}_{1}$ are in the multi-TeV regime as well-have been suggested by Brümmer and Buchmüller [161]. We will discuss one example in Sec. IV H.

\section{NUHM2 benchmark with light $A, H$, and $H^{ \pm}$}

This benchmark point, constructed within the 2parameter nonuniversal Higgs model (NUHM2), provides a model with relatively light $A, H$, and $H^{ \pm}$Higgs bosons while the remaining sparticles are beyond current LHC reach. We adopt parameters $m_{0}=10 \mathrm{TeV}, m_{1 / 2}=$ $0.5 \mathrm{TeV}, A_{0}=-16 \mathrm{TeV}, \tan \beta=7$ with $\mu=6 \mathrm{TeV}$ and $m_{A}=275 \mathrm{GeV}$. With such a light $H^{+}$, then $t H^{+}$loop contributions to $B F(b \rightarrow s \gamma)$ are large and nonminimal flavor violation in the $b$ sector would be needed.

The resulting mass spectrum is listed in Table I and displayed in Fig. 4 for all sparticles (left), and for masses below $500 \mathrm{GeV}$ only (right).

The only colored sparticles accessible to the LHC are the gluinos with $m_{\tilde{g}}=1.46 \mathrm{TeV}$, while most squarks live at around $m_{\tilde{q}} \simeq 10 \mathrm{TeV}$. The gluino decays are dominated by $\tilde{g} \rightarrow \tilde{\chi}_{1}^{0} t \bar{t}$ and $\tilde{g} \rightarrow\left(\tilde{\chi}_{1}^{ \pm} \rightarrow \tilde{\chi}_{1}^{0} W^{ \pm}\right) t b$, and thus will require dedicated analyses for high multiplicity final states or

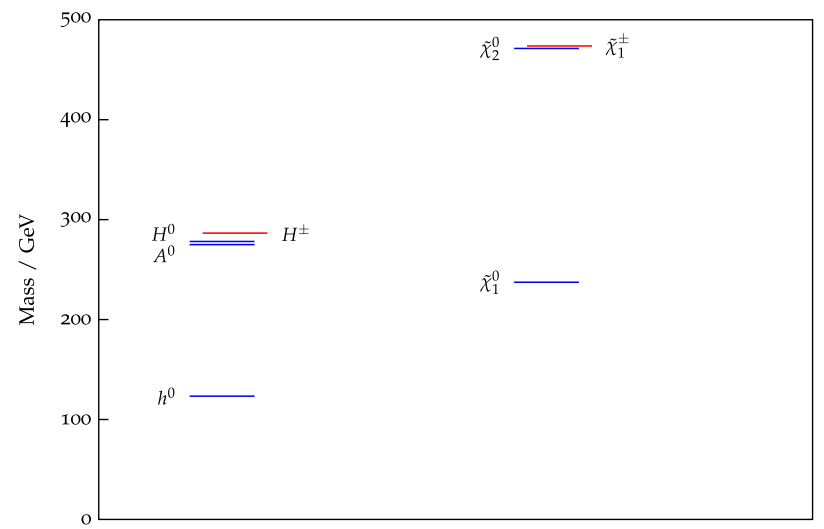

FIG. 4 (color online). Left: Full spectrum of the NUHM2 benchmark. Right: Zoom into the spectrum below 500 GeV. 
boosted techniques for identifying $W$ or $t$ jets. The signal $p p \rightarrow \tilde{\chi}_{1}^{+} \tilde{\chi}_{2}^{0} \rightarrow W h+E_{T}^{\text {miss }} \rightarrow \ell \nu_{\ell}+b \bar{b}+E_{T}^{\text {miss }}$ should ultimately be observable at LHC14 [162]. The Higgs bosons, apart from the light $C P$-even one, can most probably not be observed at the LHC in this low $\tan \beta$ and $m_{A}$ region [163].

At the ILC with $\sqrt{s} \simeq 0.5 \mathrm{TeV}$, we expect $e^{+} e^{-} \rightarrow A h$, $Z H$ to occur at observable rates. As $\sqrt{s}$ rises beyond $600 \mathrm{GeV}, A H$ and $H^{+} H^{-}$production becomes accessible while mixed $\tilde{\chi}_{1}^{0} \tilde{\chi}_{2}^{0}$ pair production, though accessible, is suppressed. At $1 \mathrm{TeV}, \tilde{\chi}_{1}^{ \pm}$and $\tilde{\chi}_{2}^{0}$ pairs will be produced in addition. Because of heavy sleptons and the sizable mass gap between $\tilde{\chi}_{1}^{ \pm}, \tilde{\chi}_{2}^{0}$, and the $\tilde{\chi}_{1}^{0}$, one expects electroweakino decays to real $W^{ \pm}$and $Z$ bosons, very similar to the "Point 5" benchmark studied in the Letter of Intents of the ILC experiments [164,165].

\section{MSUGRA/CMSSM}

Large portions of mSUGRA model parameter space are now ruled out by direct searches for gluino and squark production at LHC8. In addition, if one requires $m_{h} \simeq$ 124-126 GeV, then even larger portions of parameter space are excluded: $m_{1 / 2}<1 \mathrm{TeV}$ (corresponding to $m_{\tilde{g}}<2.2 \mathrm{TeV}$ ) for low $m_{0}$ and $m_{0}<2.5 \mathrm{TeV}$ (corresponding to $m_{\tilde{q}}<2.5 \mathrm{TeV}$ ) for low $m_{1 / 2}$ [116]. These tight constraints rule out almost all of the coannihilation and $A$-funnel annihilation regions $[40,116]$. The hyperbolic branch/FP region moves to very large $m_{0} \gtrsim 10 \mathrm{TeV}$ since now $\left|A_{0}\right|$ must be large to accommodate the rather large value of $m_{h}$. Some remaining dark matter allowed parameter space thus remains.

An example is provided by an mSUGRA benchmark point with $m_{0}=10 \mathrm{TeV}, m_{1 / 2}=0.8 \mathrm{TeV}, A_{0}=-5.45 \mathrm{TeV}$ and $\tan \beta=15$. The masses are shown in Table I and in Fig. 5 for all sparticles (left), and for masses below $500 \mathrm{GeV}$ only (right).

At this point, $m_{\tilde{g}}=2130 \mathrm{GeV}$ and $m_{\tilde{q}} \simeq 10 \mathrm{TeV}$ so colored sparticles may be beyond LHC14 reach. The most promising signature for the LHC may again be $p p \rightarrow \tilde{\chi}_{1}^{+} \tilde{\chi}_{2}^{0} \rightarrow W^{*} h^{*}+E_{T}^{\text {miss }}$.

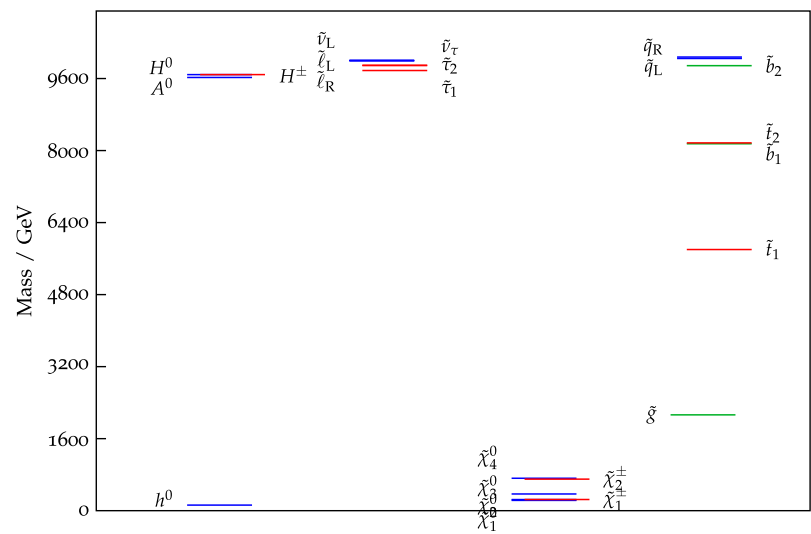

However, $\mu \simeq 234 \mathrm{GeV}$ and so $m_{\tilde{\chi}_{1}^{ \pm}}=248 \mathrm{GeV}$, $m_{\tilde{\chi}_{2}^{0}}=247 \mathrm{GeV}$ and $m_{\tilde{\chi}_{1}^{0}}=229 \mathrm{GeV}$. Thus, this pointalthough still fine-tuned in the EW sector (with $\Delta_{\mathrm{EW}}=$ 321 due to $m_{\tilde{t}_{1,2}} \simeq 6-8 \mathrm{TeV}$ )—would allow $\tilde{\chi}_{1}^{+} \tilde{\chi}_{1}^{-}$and $\tilde{\chi}_{1}^{0} \tilde{\chi}_{2}^{0}$ production at ILC with $\sqrt{s}=0.5 \mathrm{TeV}$. The $\tilde{\chi}_{1}^{0}$ would be of mixed bino-Higgsino variety and the $\tilde{\chi}_{1}^{ \pm}-\tilde{\chi}_{1}^{0}$ mass gap is just $19 \mathrm{GeV}$. When increasing $\sqrt{s}$ towards $1 \mathrm{TeV}$, the heavier neutralinos become accessible in mixed production and $\tilde{\chi}_{3}^{0}$ pair production.

\section{E. Nonuniversal gaugino masses (NUGM)}

In supergravity, gaugino masses arise from the Lagrangian term (using four-component spinor notation)

$$
\mathcal{L}_{F}^{G}=-\left.\frac{1}{4} e^{G / 2} \frac{\partial f_{A B}^{*}}{\partial \hat{h}^{* j}}\right|_{\hat{h} \rightarrow h}\left(G^{-1}\right)_{k}^{j} G^{k} \bar{\lambda}_{A} \lambda_{B}
$$

where $f_{A B}$ is the holomorphic gauge kinetic function with gauge indices $A, B$ in the adjoint representation, $\lambda_{A}$ are four-component gaugino fields and the $\hat{h}_{m}$ are hidden sector fields needed for breaking of supergravity. If $f_{A B} \sim$ $\delta_{A B}$, then gaugino masses are expected to be universal at the high energy scale where SUSY breaking takes place. However, in general supergravity, $f_{A B}$ need only transform as the symmetric product of two adjoints. In general, gaugino masses need not be universal at any energy scale, giving rise to models with nonuniversal gaugino masses (NUGM).

For a NUGM benchmark, we select a model with $m_{0}=3 \mathrm{TeV}, A_{0}=-6 \mathrm{TeV}, \tan \beta=25$ and $\mu>0$. We select gaugino masses at the GUT scale as $M_{1}=0.3 \mathrm{TeV}$, $M_{2}=0.25 \mathrm{TeV}$, and $M_{3}=0.75 \mathrm{TeV}$. The spectrum is listed in column 6 of Table I and displayed in Fig. 6 for all sparticles (left), and for masses below $500 \mathrm{GeV}$ only (right).

With $m_{\tilde{g}} \simeq 1.8 \mathrm{TeV}$ and $m_{\tilde{q}} \simeq 3 \mathrm{TeV}$, the model is clearly beyond LHC8 reach for gluinos and squarks. The model should be testable in future LHC searches, not only in the standard jets plus missing $E_{t}$ analyses, but also via

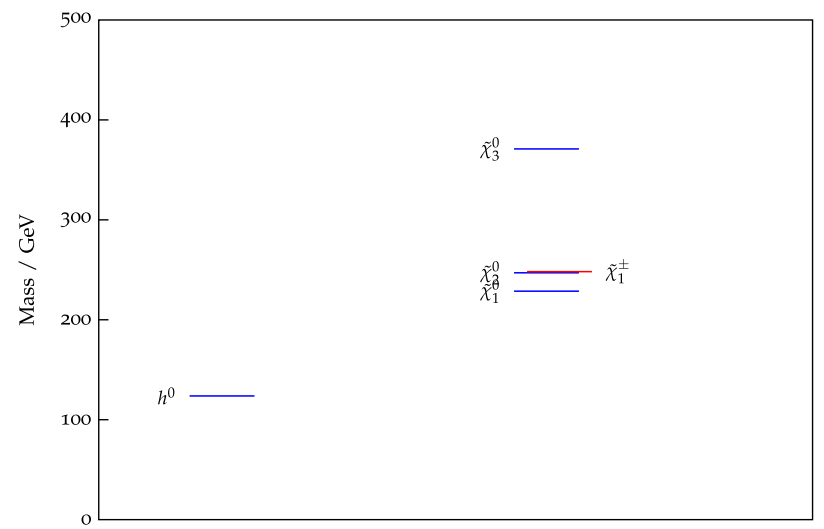

FIG. 5 (color online). Left: Full spectrum of the mSugra benchmark. Right: Zoom into the spectrum below $500 \mathrm{GeV}$. 

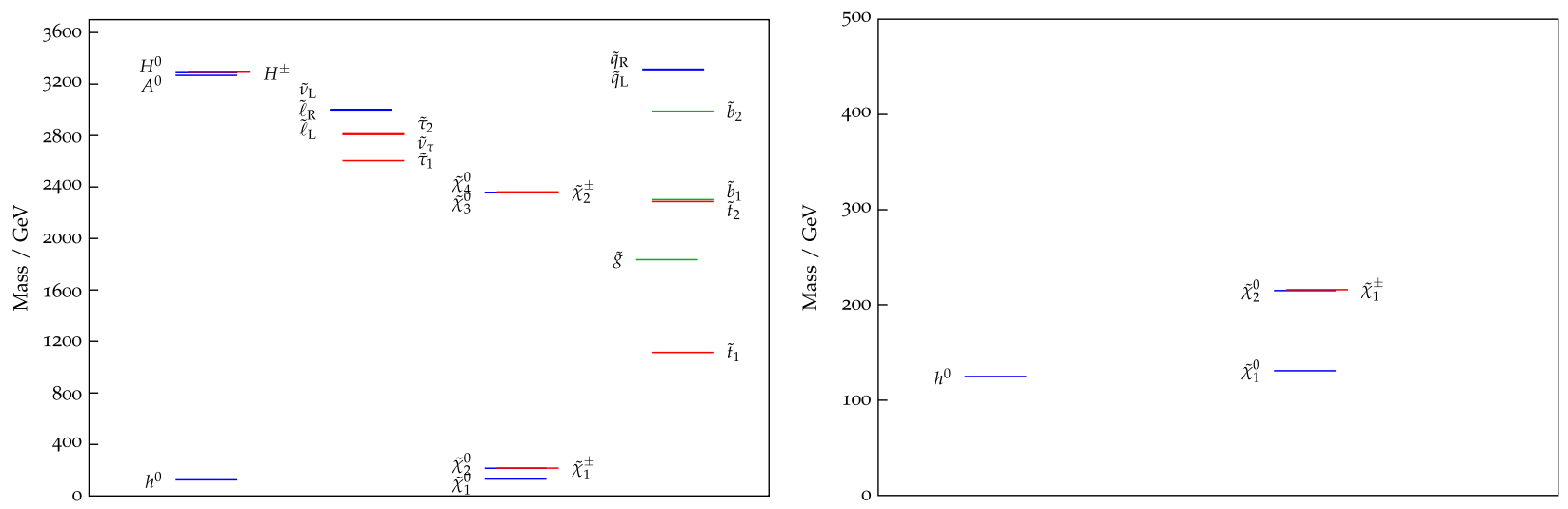

FIG. 6 (color online). Left: Full spectrum of the NUGM benchmark. Right: Zoom into the spectrum below 500 GeV.

searches tailored for very high multiplicity final states and using $b$-jet tagging [166], since the gluino almost exclusively decays via $\tilde{g} \rightarrow \tilde{t}_{1} t$ followed by $\tilde{t}_{1} \rightarrow \tilde{\chi}_{1}^{0} t$. In addition, the production channel $p p \rightarrow \tilde{\chi}_{1}^{ \pm} \tilde{\chi}_{2}^{0} \rightarrow W Z+$ $E_{T}^{\text {miss }}$ may be testable in the near future [167].

The rather light spectrum of electroweak gauginos with $m_{\tilde{\chi}_{1}^{ \pm}} \simeq 1.6 m_{\tilde{\chi}_{1}^{0}} \simeq 216 \mathrm{GeV}$ allows for chargino pair production at ILC followed by $\tilde{\chi}_{1}^{ \pm} \rightarrow \tilde{\chi}_{1}^{0} W$ decay, yielding a $W^{+} W^{-}+\not E$ signature. The $\tilde{\chi}_{1}^{0} \tilde{\chi}_{2}^{0}$ and $\tilde{\chi}_{2}^{0} \tilde{\chi}_{2}^{0}$ production channels tend to be suppressed, but may offer additional search avenues albeit at low rates.

\section{F. A $\tilde{\tau}$-coannihilation scenario}

In many constrained SUSY models where slepton and squark masses are correlated at some high energy scale, relatively light sleptons with mass $\sim 100-200 \mathrm{GeV}$ are forbidden. However, if we invoke the greater parameter freedom of the pMSSM, then spectra with light sleptons and heavy squarks can easily be generated. In fact, these models have a motivation in that they offer efficient dark matter annihilation mechanisms if the $\tilde{\tau}_{1}$ is light enough, while they at the same time naturally reconcile the measured $(g-2)_{\mu}$ anomaly (which favors light smuons) with the measured $b \rightarrow s \gamma$ branching fraction (which favors rather heavy third generation squarks).

In the pMSSM $[168,169]$, one inputs weak scale values of the following parameters: (1) $m_{\tilde{g}}, \mu, m_{A}, \tan \beta$; (2) $m_{Q}$, $m_{U}, m_{D}, m_{L}, m_{E}$ for each of the three generations; (3) gaugino masses $M_{1}$ and $M_{2}$; and (4) third generation trilinears $A_{t}, A_{b}$, and $A_{\tau}$. This gives a 19 dimensional parameter space if first and second generation scalar masses are taken as degenerate, or else a 24 dimensional parameter space for independent first, second, and third generations. ${ }^{5}$ As an example, we specify the "STC" benchmark with the following parameters, all given at a scale of $1 \mathrm{TeV}$ :

\footnotetext{
${ }^{5}$ Alternatively, the $S U(3)$ gaugino mass $M_{3}$ may be substituted for the physical gluino mass as an input.
}

(i) Higgs sector parameters: $\tan (\beta)=10, \mu=400 \mathrm{GeV}$, $m_{A}=400 \mathrm{GeV}$,

(ii) trilinear couplings: $A_{t}=A_{b}=A_{\tau}=-2.1 \mathrm{TeV}$,

(iii) gaugino mass parameters: $M_{3}=2 \mathrm{TeV}$, $M_{2}=210 \mathrm{GeV}$ (yields $M_{1}=100 \mathrm{GeV}$ via GUT relation),

(iv) slepton mass parameters: $m_{L}(1,2,3)=205 \mathrm{GeV}$, $m_{E}(1,2,3)=117.5 \mathrm{GeV}$

(v) squark mass parameters: $m_{Q}(1,2)=m_{D}(1,2)=$ $m_{U}(1,2)=2 \mathrm{TeV}, \quad m_{Q}(3)=1.5 \mathrm{TeV}, \quad m_{U}(3)=$ $500 \mathrm{GeV}, m_{D}(3)=800 \mathrm{GeV}$.

Since $M_{1}$ and $M_{2}$ follow the GUT relation, there are effectively 12 independently chosen parameters. The resulting sparticle masses, which have been obtained with SPHENO [170,171], along with low energy and cosmic observables obtained from MICROMEGAS [172], are listed in Table II and displayed in Fig. 7 for all sparticles (left), and for masses below $500 \mathrm{GeV}$ only (right).

The total SUSY cross section at the LHC is about $1.5 \mathrm{pb}$ at $8 \mathrm{TeV}$ and doubles to $3 \mathrm{pb}$ at $14 \mathrm{TeV}$. The total SUSY cross section is dominated by electroweakino production, which contributes about two thirds of the rate, followed by $\tilde{t}_{1}$ pair production. The cross section for light squark production is a few fb. Thus, with masses around $2 \mathrm{TeV}$, the gluino and the partners of the light quarks are beyond current LHC limits, also since the gluino decays dominantly via $\tilde{t}_{1} t$ or $\tilde{b}_{1} b$. Although light sleptons are present, the current limits on direct electroweakino production [173] do not cover this case due to the small mass difference between the $\tilde{\tau}_{1}$ and the $\tilde{\chi}_{1}^{0}$, which leads to soft $\tau$ leptons in the chargino and neutralino decays instead of the searched for high $p_{t}$ electrons and muons.

At LHC14, a clear deviation from the SM would be observed in this scenario, but it is subject to future study which of the numerous contributing sparticles could be identified.

At the ILC, all sleptons and the lighter electroweakinos would be observable $\sqrt{s} \lesssim 500 \mathrm{GeV}$. Especially the selectron pair production cross section would be huge. In addition, the light top squarks as well as the heavy Higgs bosons and 
TABLE II. Mass spectrum and rates for post-LHC8 benchmark points 6-10. All masses and dimensionful parameters are in TeV units. All values are obtained from ISASUGRA for KL and NMH, while for STC, BB, and LMH SPheno, SOFTSUSY and MICROMEGAS have been used.

\begin{tabular}{|c|c|c|c|c|c|}
\hline Mass & STC & KL & BB & $\mathrm{NMH}$ & LMH \\
\hline $\tan \beta$ & 10 & 10 & 48 & 20 & 6.2 \\
\hline$\mu$ & 0.4 & 2.0 & 0.160 & 3.1 & 1.7 \\
\hline$m_{A}$ & 0.400 & 2.0 & 4.05 & 5.36 & 0.110 \\
\hline$m_{h}$ & 0.124 & 0.125 & 0.127 & 0.125 & 0.103 \\
\hline$m_{H}$ & 0.401 & 2.013 & 4.04 & 5.34 & 0.127 \\
\hline$m_{H^{ \pm}}$ & 0.408 & 2.014 & 4.05 & 5.40 & 0.134 \\
\hline$m_{\tilde{g}}$ & 2.0 & 1.79 & 9.91 & 1.77 & 1.58 \\
\hline$m_{\tilde{\chi}_{12}^{ \pm}}^{0}$ & $0.206,0.425$ & $0.200,2.05$ & $0.167,9.52$ & $0.535,3.1$ & $0.211,1.7$ \\
\hline$m_{\tilde{\chi}_{1,2}^{0}}$ & $0.096,0.206$ & $0.200,0.616$ & $0.167,0.168$ & $0.246,0.533$ & $0.095,0.211$ \\
\hline$m_{\tilde{\chi}_{3,4}^{0,2}}^{0}$ & $0.408,0.424$ & $2.05,2.05$ & $5.30,9.51$ & $3.06,3.07$ & $1.70,1.70$ \\
\hline$m_{\tilde{u}_{L, R}, 4}$ & $2.0,2.03$ & $24.8,25.3$ & $11.1,8.87$ & $1.62,1.61$ & $1.54,1.54$ \\
\hline$m_{\tilde{t}_{1,2}}$ & $0.416,1.53$ & $16.4,20.9$ & $6.25,9.57$ & $2.07,3.82$ & $1.34,1.64$ \\
\hline$m_{\tilde{d}_{L, R}}$ & $2.03,2.03$ & $24.8,24.8$ & $11.1,8.49$ & $1.62,1.16$ & $1.54,1.54$ \\
\hline$m_{\tilde{b}_{1,2}}$ & $0.795,1.51$ & $20.8,24.7$ & $6.90,9.57$ & $3.84,4.93$ & $1.51,1.54$ \\
\hline$m_{\tilde{e}_{L, R}}$ & $0.213,0.128$ & $25.3,24.4$ & $7.73,4.60$ & $0.511,0.252$ & $1.50,1.50$ \\
\hline$m_{\tilde{\tau}_{1,2}}^{L, n}$ & $0.107,0.219$ & $24.3,25.2$ & $2.87,7.30$ & $4.65,4.85$ & $0.126,1.50$ \\
\hline$\Omega_{\tilde{\chi}^{0}}^{\text {std }} h^{2}$ & 0.115 & 0.0025 & 0.003 & 0.12 & 0.115 \\
\hline$\langle\stackrel{x}{\sigma v}\rangle \times 10^{25}\left[\mathrm{~cm}^{3} / \mathrm{s}\right]$ & 0.021 & 19 & 2.9 & 0.001 & 0.000012 \\
\hline$\sigma^{\mathrm{SI}}\left(\tilde{\chi}^{0} p\right) \times 10^{9}[\mathrm{pb}]$ & 1.12 & 0.04 & 0.013 & 0.0005 & 9.3 \\
\hline$a_{\mu}^{\mathrm{SUSY}} \times 10^{10}$ & 25.6 & 0.0002 & 0.008 & 25.6 & 0.43 \\
\hline$B F(b \rightarrow s \gamma) \times 10^{4}$ & 3.2 & 3.2 & 3.3 & 3.2 & 5.2 \\
\hline$B F\left(B_{s} \rightarrow \mu \bar{\mu}\right) \times 10^{9}$ & 3.4 & 3.8 & 3.1 & 3.8 & 4.4 \\
\hline$B F\left(B_{u} \rightarrow \tau \nu_{\tau}\right) \times 10^{4}$ & 1.1 & 1.3 & 1.2 & 1.3 & 1.1 \\
\hline$\Delta_{\mathrm{EW}}$ & 38 & 962 & 275 & 2263 & 710 \\
\hline$\Delta_{\mathrm{HS}}$ & 38 & $1.3 \times 10^{5}$ & 1073 & 8295 & 710 \\
\hline
\end{tabular}

heavy electroweakinos would be accessible at ILC with $\sqrt{s} \simeq$ $1 \mathrm{TeV}$. It has be shown in a similar scenario that masses and cross sections in the stau sector, as well as the stau polarization, can be measured to a few percent at less [174].

Because of the large number of production processes open already at $\sqrt{s} \simeq 500 \mathrm{GeV}$, which often yield long cascades [175], STC is actually an experimentally challenging scenario for ILC. Therefore, it is an ideal case study to demonstrate the separation of many closely spaced new matter states with all the tools offered by ILC, including threshold scans and different beam polarization configurations, but also taking into account realistic assumptions on the beam energy spectrum, accelerator backgrounds, and detector resolutions.

At a center-of-mass energy of $1 \mathrm{TeV}$ or above, the separation of the heavier electroweakinos as well as of the nearby $\tilde{t}_{1}$ and the heavy Higgs states will provide additional experimental challenges.
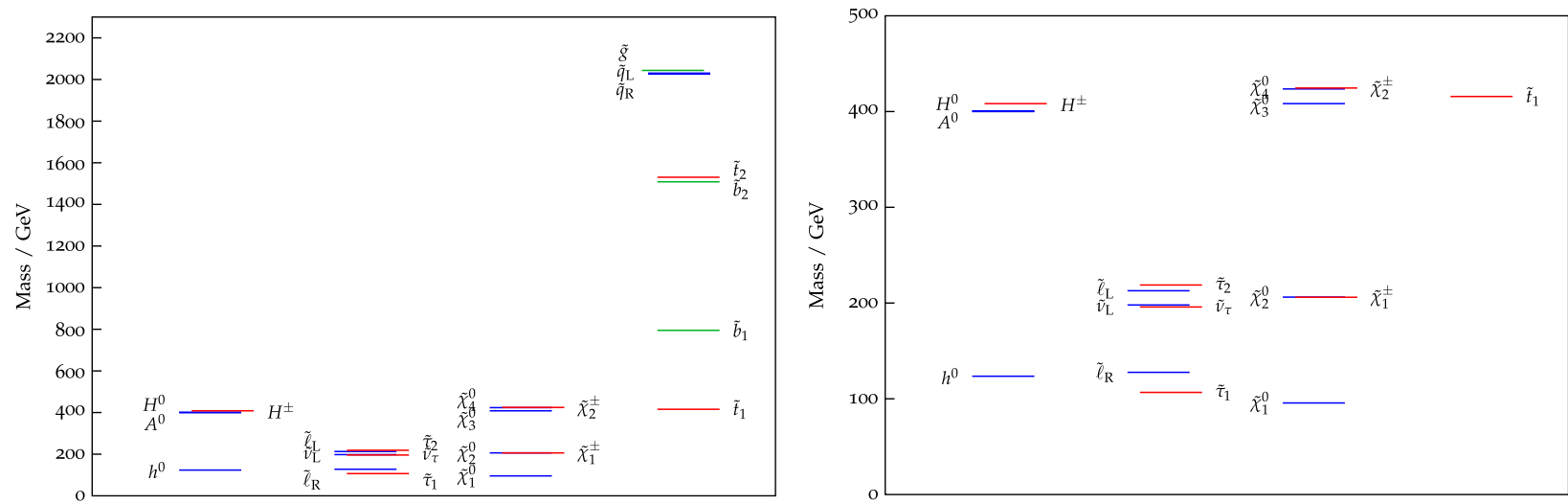

FIG. 7 (color online). Left: Full spectrum of the STC benchmark. Right: Zoom into the spectrum below $500 \mathrm{GeV}$. 


\section{G. Kallosh-Linde (KL), G2MSSM, spread SUSY benchmark}

While minimal anomaly mediation seems on shaky ground due to its prediction of a light Higgs scalar $m_{h} \lesssim$ $120 \mathrm{GeV}$, other similar models have emerged as perhaps more compelling. One of these models-by Kallosh and Linde (the KL model [176,177])—attempts to stabilize stringy moduli fields via a generalization of the KKLT method [178] utilizing a racetrack superpotential. The moduli in this theory end up superheavy and allow for the chaotic inflationary scenario to emerge in supergravity models. In this class of models, the various scalar fields have a mass of the order of the gravitino mass, with $m_{3 / 2} \simeq$ $100 \mathrm{TeV}$. The gauginos, however, remain below the $\mathrm{TeV}$ scale, and adopt the usual AMSB form. This model is also typical of "spread supersymmetry" [179]. Another stringy model by Acharya et al. [180] known as G2MSSM also predicts multi-TeV scalars. In the G2MSSM, the gauginos are again light, typically with $M_{2} \ll M_{1} \simeq M_{3}$ so that again a model with light wino-like $\tilde{\chi}_{1}^{ \pm}$and $\tilde{\chi}_{1}^{0}$ emerges.

To model these cases, we adopt the NUHM2 model, but with nonuniversal gaugino masses, with parameters chosen as $m_{0}=25 \mathrm{TeV}, m_{1 / 2}=200 \mathrm{GeV}, A_{0}=0, \tan \beta=10$ with $\mu=m_{A}=2 \mathrm{TeV}$. We then set GUT-scale gaugino masses to the AMSB form given by $M_{1}=1320 \mathrm{GeV}$, $M_{2}=200 \mathrm{GeV}$, and $M_{3}=-600 \mathrm{GeV}$.

The spectrum is listed in Table II and displayed in Fig. 8 for all sparticles (left), and for masses below $500 \mathrm{GeV}$ only (right). The wino-like $\tilde{\chi}_{1}^{0}$ state is the lightest MSSM particle with mass $m_{\tilde{\chi}_{1}^{0}}=200.07 \mathrm{GeV}$ while the wino-like lightest chargino has mass $m_{\tilde{\chi}_{1}^{ \pm}}=200.4 \mathrm{GeV}$. We also have a bino-like $\tilde{\chi}_{2}^{0}$ with $m_{\tilde{\chi}_{2}^{0}}=616.5 \mathrm{GeV}$ and a gluino with $m_{\tilde{g}}=1788 \mathrm{GeV}$. All matter scalars have mass near the $25 \mathrm{TeV}$ scale, and so decouple. The light Higgs scalar has mass $m_{h}=125 \mathrm{GeV}$.

In this case, gluino pair production may barely be accessible to LHC14 with order $100 \mathrm{fb}^{-1}$ of data [181]. At ILC, the decay products from chargino decay will be extremely soft. However, the wino-like chargino is then quasistable, flying of order centimeters before decay, leaving a highly ionizing track (HIT) which terminates upon decay into very soft decay products. Chargino pair production could be revealed at ILC via initial state radiation of a hard photon, and then identification of one or more HITs, or stubs. In addition, if $\sqrt{s}$ is increased to $\sim 1 \mathrm{TeV}$, then $\tilde{\chi}_{1}^{0} \tilde{\chi}_{2}^{0}$ production opens up, although rates are expected to be small. In this case, one expects $\tilde{\chi}_{2}^{0} \rightarrow W \tilde{\chi}_{1}^{ \pm}$or $\tilde{\chi}_{1}^{0} h$ to occur.

\section{H. Brümmer-Buchmüller (BB) benchmark}

Brümmer and Buchmüller have proposed a model wherein the Fermi scale emerges as a focus point within high scale gauge mediation [161]. The model is inspired by GUT-scale string compactifications which frequently predict a large number of vectorlike states in incomplete GUT multiplets which may serve as messenger fields for gauge mediated SUSY breaking which is implemented at or around the GUT scale. By adopting models with large numbers of messenger fields, it is found that the weak scale emerges quite naturally from the scalar potential as a focus point from renormalization group equation running of the soft terms. The soft SUSY breaking terms receive both gauge-mediated and gravity-mediated contributions. The gauge-mediated contributions are dominant for most soft masses, while the $A$ terms and $\mu$ may be forbidden by symmetry. The superpotential Higgsino mass term $\mu$ emerges from gravitational interactions and is expected to be of the order of the gravitino mass $\mu \simeq m_{3 / 2} \simeq$ $150-200 \mathrm{GeV}$. The spectrum which emerges from the model tends to contain gluino and squark masses in the several $\mathrm{TeV}$ range so that the model is compatible with LHC constraints. States accessible to a linear collider would include the Higgsino-like light charginos $\tilde{\chi}_{1}^{ \pm}$and neutralinos $\tilde{\chi}_{1,2}^{0}$.

For ILC studies, we adopt the benchmark model with messenger indices $\left(N_{1}, N_{2}, N_{3}\right)=(46,46,20)$, a characteristic gauge-mediated soft mass per messenger pair $m_{\mathrm{GM}}=250 \mathrm{GeV}, \tan \beta=48$ and weak scale values of
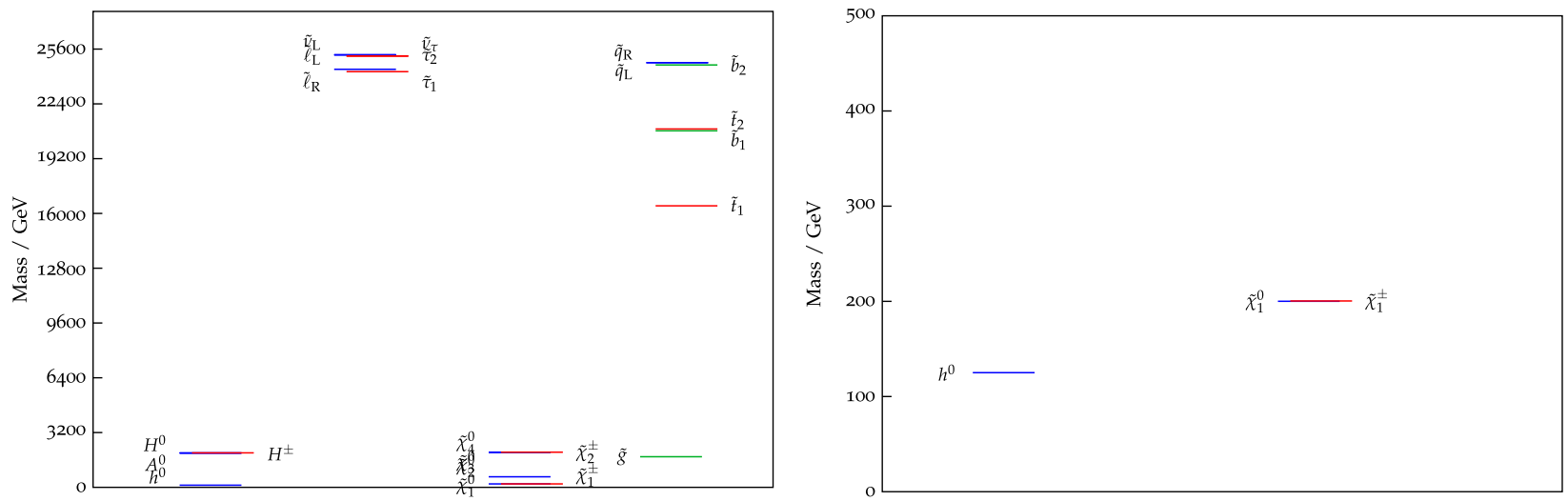

FIG. 8 (color online). Left: Full spectrum of the KL benchmark. Right: Zoom into the spectrum below $500 \mathrm{GeV}$. 

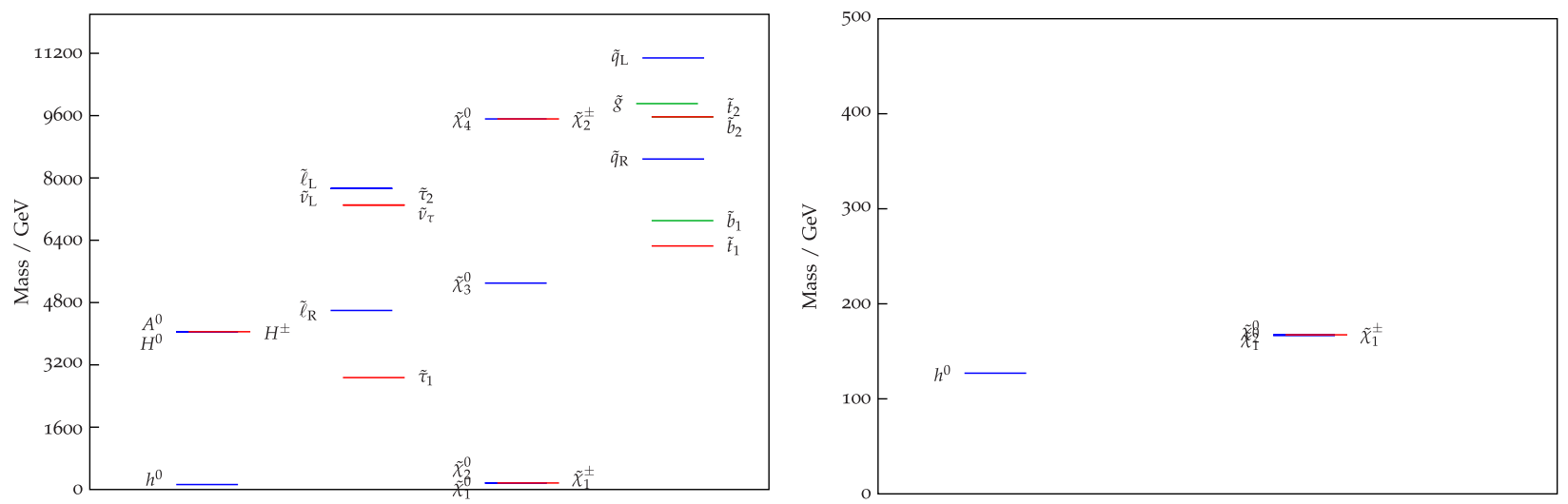

FIG. 9 (color online). Left: Full spectrum of the BB benchmark. Right: Zoom into the spectrum below $500 \mathrm{GeV}$.

$\mu=167 \mathrm{GeV}$ and $m_{A}=4.05 \mathrm{TeV}$. This results in a spectrum which is mostly on the multi-TeV scale, with only the four Higgsinos in collider range. The relevant lowscale gaugino mass parameters are $M_{1}=5.3 \mathrm{TeV}$ and $M_{2}=9.5 \mathrm{TeV}$.

The spectrum, generated from SOFTSUSY [182], is listed in Table II, along with low energy and cosmic observables obtained from MICROMEGAS [172]. ${ }^{6}$ Figure 9 displays the masses for all sparticles (left), and for masses below $500 \mathrm{GeV}$ only (right).

Although the mass splitting between the Higgsinos is below $1 \mathrm{GeV}$, they can be observed at the ILC and their masses and cross sections measured at the percent level. This has recently been demonstrated in a simulation study [184] based on the ILD detector concept, including in particular the effect of beam background on the reconstruction of low-momentum charged particles. The obtained resolutions allow one to determine the $\mu$ parameter within a $3 \mathrm{GeV}$ window $(1 \sigma)$. The bounds on $M_{1}$ and $M_{2}$ are strongly correlated, but in the $M_{1}-M_{2}$ plane, both parameters can be constrained to a narrow band in the multi-TeV regime.

\section{Normal scalar mass hierarchy (NMH)}

Models with a normal scalar mass hierarchy $\left[m_{0}(1) \simeq\right.$ $\left.m_{0}(2) \ll m_{0}(3)\right]$ [185] are motivated by the attempt to reconcile the $>3 \sigma$ discrepancy in $(g-2)_{\mu}$ (which requires rather light sub-TeV smuons) with the lack of a large discrepancy in $B F(b \rightarrow s \gamma)$, which seems to require third generation squarks beyond the $\mathrm{TeV}$ scale. The idea here is to require a high degree of degeneracy amongst first/second generation sfermions in order to suppress the most stringent flavor changing neutral current processes, while allowing third generation sfermions to be highly split, since flavor changing neutral current constraints from third generation particles are relatively mild. The normal mass hierarchy follows in that first/second generation scalars are assumed

\footnotetext{
${ }^{6} \mathrm{~A}$ previous - somewhat different-version of the BB benchmark point generated from Isasugra is located in Ref. [183].
}

much lighter than third generation scalars, at least at the GUT scale. Renormalization group running then lifts first/ second generation squark masses to high values such that $m_{\tilde{q}} \simeq m_{\tilde{g}}$. However, first/second generation sleptons remain in the several hundred $\mathrm{GeV}$ range since they have no strong coupling.

Here, we adopt a NMH benchmark point with independent scalar and gaugino masses at the GUT scale. We adopt the following parameters: $m_{5}(3) \simeq m_{10}(3)=5 \mathrm{TeV}$, $m_{1 / 2}=0.63 \mathrm{TeV}, A_{0}=-8.5 \mathrm{TeV}, \tan \beta=20, \mu>0$ with $m_{L}(1,2)=0.21 \mathrm{TeV}, m_{E}(1,2)=0.387 \mathrm{TeV}$, and $m_{Q}(1,2)=m_{U}(1,2)=m_{D}(1,2) \equiv m_{10}(1,2)=0.8 \mathrm{TeV}$. We also take GUT-scale gaugino masses as $M_{1}=$ $0.56 \mathrm{TeV}, M_{2}=0.63 \mathrm{TeV}$, and $M_{3}=0.75 \mathrm{TeV}$.

The spectrum generated using ISASUGRA 7.83 with nonuniversal soft terms is listed in Table II and displayed in Fig. 10 for all sparticles (left), and for masses below $500 \mathrm{GeV}$ only (right). ${ }^{7}$

We find $m_{\tilde{\chi}_{1}^{0}} \simeq 246 \mathrm{GeV}, \quad m_{\tilde{e}_{R}} \simeq m_{\tilde{\mu}_{R}}=252 \mathrm{GeV}$, $m_{\tilde{\nu}_{e, \mu L}} \simeq 302 \mathrm{GeV}$, and $m_{\tilde{e}_{L}} \simeq m_{\tilde{\mu}_{L}}=512 \mathrm{GeV}$, as well as $m_{h} \simeq 125 \mathrm{GeV}$. In the colored sector, $m_{\tilde{g}}=1.77 \mathrm{TeV}$ with $m_{\tilde{q}} \simeq 1.6 \mathrm{TeV}$, so the model is compatible with LHC8 constraints, but should be testable at LHC14. The first and second generation squarks decay mainly into $\tilde{\chi}_{1}^{ \pm}+$jet, followed by $\tilde{\chi}_{1}^{ \pm} \rightarrow \tilde{\nu}_{l} l \rightarrow \tilde{\chi}_{1}^{0} \nu_{l} l$, or alternatively into $\tilde{\chi}_{2}^{0}+$ jet, followed by $\tilde{\chi}_{2}^{0} \rightarrow \tilde{\nu}_{l} \nu_{l} \rightarrow \tilde{\chi}_{1}^{0} \nu_{l} \nu_{l}$. Thus, squark pair production will give only two jets, either accompanied by just missing transverse energy or by one or two leptons. The gluino decays mostly into first or second generation squarks plus an additional jet. Since the $\tilde{\chi}_{2}^{0}$ decays invisibly, the only sign of direct $\tilde{\chi}_{1}^{ \pm} \tilde{\chi}_{2}^{0}$ production will be a single lepton from the the $\tilde{\chi}_{1}^{ \pm}$ decay plus missing transverse energy.

The model does indeed reconcile $(g-2)_{\mu}$ with $B F(b \rightarrow s \gamma)$ since $\Delta a_{\mu}^{\mathrm{SUSY}} \simeq 26 \times 10^{-10}$ and $B F(b \rightarrow s \gamma)=$ $3.15 \times 10^{-4}$. Also, the thermal neutralino abundance is

\footnotetext{
${ }^{7} \mathrm{NMH}$ benchmark models are rather sensitive to parameter choices and care must be taken to generate $m_{h} \simeq 125 \mathrm{GeV}$ whilst respecting LHC and $b \rightarrow s \gamma$ constraints and at the same time generating large $\Delta a_{\mu}$.
} 

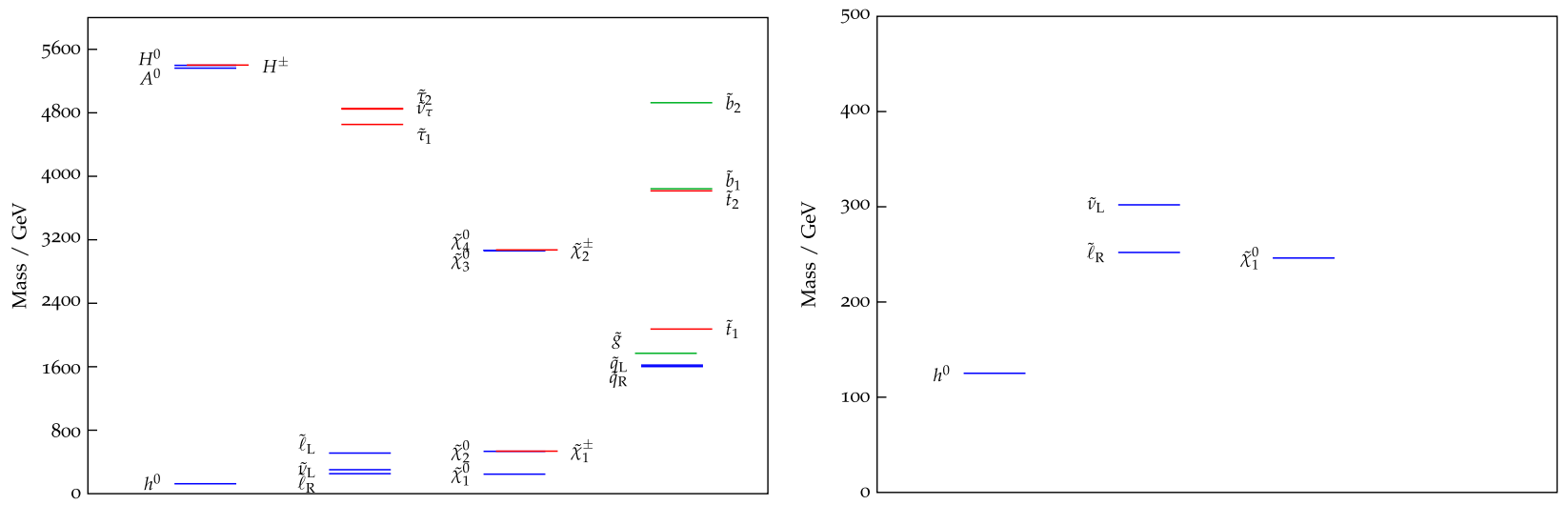

FIG. 10 (color online). Left: Full spectrum of the NMH benchmark. Right: Zoom into the spectrum below $500 \mathrm{GeV}$.

given as $\Omega_{\tilde{\chi}_{1}^{0}} h^{2} \simeq 0.11$ due to neutralino-slepton coannihilation. An ILC with $\sqrt{s} \gtrsim 500 \mathrm{GeV}$ would be needed to access the $\tilde{e}_{R} \overline{\tilde{e}}_{R}$ and $\tilde{\mu}_{R} \overline{\tilde{\mu}}_{R}$ pair production. These reactions would give rise to very low energy dielectron and dimuon final states which would be challenging to extract from two-photon backgrounds. However, since it has been demonstrated that mass differences of this size are manageable even in the case of $\tau$ leptons from $\tilde{\tau}$ decays [174], it should be feasible also in the case of electrons or muons. Since $\tilde{\nu} \rightarrow \nu+\tilde{\chi}_{1}^{0}$, sneutrinos would decay invisibly, although the reaction $e^{+} e^{-} \rightarrow$ $\tilde{\nu}_{L} \overline{\tilde{\nu}}_{L} \gamma$ may be a possibility. The lack of $\tilde{\tau}^{+} \tilde{\tau}^{-}$pair production might give a hint that nature is described by a NMH model.

\section{J. Low $m_{H}$ scenario (LMH)}

For this benchmark, we assume that the $125 \mathrm{GeV}$ particle is heavy $C P$-even Higgs boson $H$ of the MSSM. We adopt the recently proposed low $m_{H}$ benchmark scenario for the Higgs sector [186]: $m_{A}=110 \mathrm{GeV}$, $M_{\mathrm{SUSY}}=1.5 \mathrm{TeV}, \quad M_{2}=200 \mathrm{GeV}, \quad X_{t}^{\mathrm{MS}}=2.9 M_{\mathrm{SUSY}}$, $A_{b}=A_{\tau}=A_{t}, m_{\tilde{g}}=1.5 \mathrm{TeV}, M_{\tilde{l}_{3}}=1 \mathrm{TeV}$. $\tan \beta$ and $\mu$ are free parameters in [186], whereas we select $\tan \beta=6.2$ and $\mu=1.7 \mathrm{TeV}$, which produces rates for the $H$ of at least $90 \%$ of SM expectations. The full mass spectrum is shown in Table II and illustrated in the left panel of Fig. 11, while the right panel is again restricted to sparticles with masses below $500 \mathrm{GeV}$.

Since the bino-like LSP annihilates only inefficiently, we lowered $M_{\tilde{l}_{3}}$ from $1 \mathrm{TeV}$ to $113 \mathrm{GeV}$. As a result, the $\tilde{\nu}_{\tau}$ becomes the NLSP with $m_{\tilde{\nu}_{\tau}}=101 \mathrm{GeV}$ followed by the $\tilde{\tau}_{1}$ with $m_{\tilde{\tau}_{1}}=126 \mathrm{GeV}$. Both of them contribute to a sufficiently high coannihilation fraction and lower the relic density to the observed value. The $\tilde{\tau}_{1}$ decays in $100 \%$ of the cases into $\tau \tilde{\chi}_{1}^{0}$.

In this channel, it has been shown that the stau mass and pair-production cross section can be measured very precisely, even in the more challenging situation of a smaller mass difference [174]. The $\tilde{\nu}_{\tau}$ however decays invisibly to $\nu_{\tau} \tilde{X}_{1}^{0}$. Since the cross section is too low for detecting an excess in the monophoton signature over the SM neutrino and SUSY $\tilde{\chi}_{1}^{0}$ pair production, it would be interesting to study if the precision on $m_{\tilde{\nu}_{\tau}}$ expected from cascade decays, especially from $\tilde{\chi}_{1}^{ \pm} \rightarrow \tilde{\nu}_{\tau} \tau$ and
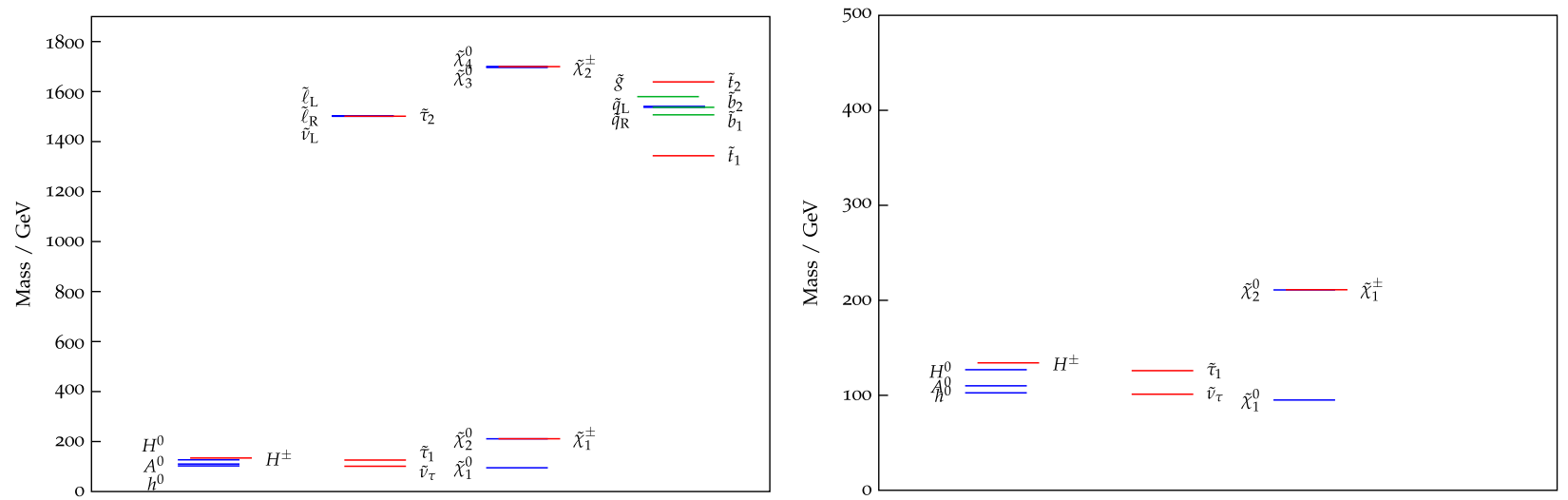

FIG. 11 (color online). Left: Full spectrum of the low MH benchmark. Right: Zoom into the spectrum below 500 GeV. 
$\tilde{\chi}_{2}^{0} \rightarrow \tilde{\nu}_{\tau} \nu_{\tau}$, allows one to predict the relic density with precisions comparable to the cosmological measurements.

The light $C P$-even Higgs has a mass of $103 \mathrm{GeV}$; this is compatible with LEP bounds since it is non-SM-like but has reduced couplings to the $Z$ boson and thus the production cross section is smaller by about a factor 10 . The $C P$-odd Higgs and the charged Higgses have masses of $m_{A}=110 \mathrm{GeV}$ and $m_{H^{ \pm}}=134 \mathrm{GeV}$. Thus, all Higgs bosons could be precisely studied at the ILC, while due to the low value of $\tan \beta$, they are difficult to observe at the LHC despite their low masses.

\section{CONCLUSIONS}

At first sight, it may appear very disconcerting that after one full year of data taking at LHC8, with $\sim 20 \mathrm{fb}^{-1}$ per experiment, no sign of supersymmetry is yet in sight. On the other hand, the discovery of a light Higgs scalar with mass $m_{h} \simeq 125 \mathrm{GeV}$ lends indirect support to SUSY: while $m_{h}$ can theoretically inhabit a rather large range of values of up to $800 \mathrm{GeV}$ in the standard model, the simplest supersymmetric extensions of the SM require it to lie below $\sim 135 \mathrm{GeV}$. A light SUSY Higgs of mass $\sim 125 \mathrm{GeV}$ seems to require top squark masses $m_{\tilde{t}_{i}} \gtrsim$ $1 \mathrm{TeV}$ along with large mixing: thus, the emerging overall view of the SUSY landscape seems more consistent with a super-TeV sparticle mass spectrum than with a sub-TeV spectrum, at least as far as gluinos and squarks are concerned. In addition, the $125 \mathrm{GeV}$ Higgs signal puts a high degree of stress, as measured by SUSY naturalness, on many popular constrained models such as CMSSM, mAMSB, and mGMSB. In the case of the MSSM, the Higgs signal may favor gravity-mediated SUSY breaking models since these naturally accommodate large mixing in the top squark sector.

While some pre-LHC analyses [based on global fits of SUSY to a variety of data which may have been overly skewed by the $(g-2)_{\mu}$ anomaly] had predicted a very light sparticle mass spectrum, the presence of a multi-TeV spectrum of at least first/second generation matter scalars was not unanticipated by many theorists. The basis of this latter statement rests on the fact that a decoupling of first/second generation matter scalars either solves or at least greatly ameliorates: the SUSY flavor problem, the SUSY $C P$ problem, the SUSY GUT proton decay problem and-in the context of gravity mediation where the gravitino mass sets the scale for the most massive SUSY particles-the gravitino problem.

Such a decoupling spectrum need not be inconsistent with electroweak fine-tuning arguments. Minimization of large log contributions to $m_{h}$ implies a SUSY spectrum including three third generation squarks with mass less than about $500 \mathrm{GeV}$ : these are the so-called natural SUSY models. While intriguing, such a spectrum seems phenomenologically disfavored by the $B F(b \rightarrow s \gamma)$, by the rather high value of $m_{h}$, and by lack of top/bottom squark signals at LHC8. On the theory side, minimization of large logs may be too harsh a fine-tuning restriction since it neglects possible correlations amongst high scale parameters (as in focus point SUSY) which lead to natural cancellations leading to low $m_{H_{u}}^{2}$ at the weak scale. The more conservative electroweak fine-tuning measure implies models with: low $|\mu| \lesssim 300 \mathrm{GeV}$, third generation squarks with $m_{\tilde{t}_{1,2}, \tilde{b}_{1}} \sim 1-4 \mathrm{TeV}$ and $m_{\tilde{g}} \sim 1-5 \mathrm{TeV}$. Since first/second generation matter scalars do not enter the electroweak scalar potential, these sparticles can indeed exist in the 10-50 $\mathrm{TeV}$ regime-as required by decoupling-without affecting fine-tuning. These radiatively driven NS models allow for heavier third generation squarks while explaining how it is that the $W, Z$, and $h$ masses all lie near the $100 \mathrm{GeV}$ scale. We have presented here four different models with the required small superpotential Higgsino mass $\mu$ : NS, RNS, focus point SUSY, and a nonminimal GMSB model suggested by Brümmer and Buchmüller (BB). The spectra from these small $\mu$ models can be difficult to detect at LHC since the characteristic light Higgsinos have a highly compressed mass spectrum. In each of these cases, however, an ILC would easily discover the predicted light Higgsino states. In such cases, the ILC would be a Higgsino factory, in addition to a Higgs factory!

We also presented several benchmark models consistent with LHC and other constraints which predict some varied phenomenology. The NUHM2 point contains heavy matter scalars but with $A$ and $H$ Higgs bosons which would also be accessible to ILC. The nonuniversal gaugino mass (NUGM) model allows for chargino pair production at ILC followed by $\tilde{\chi}_{1}^{ \pm} \rightarrow W \tilde{\chi}_{1}^{0}$ decay, leading to $W^{+} W^{-}+\not E$ events. We also presented one benchmark point consistent with Kallosh-Linde/spread SUSY/ G2MSSM models. In this case, matter scalars have masses $m_{\tilde{q}, \tilde{\ell}} \simeq m_{3 / 2} \sim 25 \mathrm{TeV}$, but gaugino masses follow the AMSB pattern, with the $\tilde{\chi}_{1}^{ \pm}$and $\tilde{\chi}_{1}^{0}$ being nearly pure wino, with $m_{\tilde{\chi}_{1}^{ \pm}}-m_{\tilde{\chi}_{1}^{0}} \simeq 0.33 \mathrm{GeV}$ mass gap. If the mass gap is small enough, then charginos can fly a measurable distance before decay. It might be possible to detect $e^{+} e^{-} \rightarrow \tilde{\chi}_{1}^{+} \tilde{\chi}_{1}^{-} \gamma \rightarrow \gamma+$ soft debris including possible highly ionizing tracks which terminate into soft pions. We presented pMSSM and NMH models with light charginos and sleptons which are in accord with the $(g-2)_{\mu}$ anomaly, $m_{h} \simeq 125 \mathrm{GeV}$ and with a standard neutralino relic abundance $\Omega_{\tilde{\chi}_{1}^{0}}^{\text {std }} h^{2}=0.11$. The ILC-relevant part of the spectrum for benchmark STC is very similar to the well-studied SPS1a scenario [187] (or its variant SPS1a'). Finally, we presented the LMH benchmark where the discovered Higgs resonance could turn out to be the heavier MSSM scalar state $H$ instead of $h$.

In summary, the LHC8 run in 2012 has resulted in the spectacular discovery of a SM-like Higgs scalar at 
$125 \mathrm{GeV}$. The Higgs discovery can be regarded as an overall positive for weak scale supersymmetry in that the mass value falls squarely within the narrow predicted window predicted by the MSSM. However, so far there is no sign of SUSY particles at LHC; instead, impressive new limits on gluino and squark masses have been determined. Even so, naturalness arguments, possibly along with the muon $g-2$ anomaly, portend a rich assortment of new matter states likely accessible to the ILC, but which remain difficult to detect at LHC. We hope the benchmark models presented here provide a broad picture of the myriad possibilities for SUSY physics which may be expected at ILC and also LHC in the post-LHC8 era.

\section{ACKNOWLEDGMENTS}

We would like to thank Mikael Berggren, Azar Mustafayev, Krzysztof Rolbiecki, and Annika Vauth for supporting calculations and valuable discussions, and Benno List and Xerxes Tata for comments on the manuscript. We thankfully acknowledge the support of the DFG through Grant No. SFB 676/2-2010.
[1] J. Wess and B. Zumino, Phys. Lett. 49B, 52 (1974).

[2] A. Salam and J. A. Strathdee, Phys. Rev. D 11, 1521 (1975).

[3] A. Salam and J. A. Strathdee, Phys. Lett. 51B, 353 (1974).

[4] For a review, see, e.g., H. Baer and X. Tata, Weak Scale Supersymmetry: From Superfields to Scattering Events (Cambridge University Press, Cambridge, England, 2006), p. 537.

[5] E. Witten, Nucl. Phys. B188, 513 (1981).

[6] R. K. Kaul, Phys. Lett. 109B, 19 (1982).

[7] S. Ferrara, D.Z. Freedman, P. van Nieuwenhuizen, P. Breitenlohner, F. Gliozzi, and J. Scherk, Phys. Rev. D 15, 1013 (1977).

[8] E. Cremmer, S. Ferrara, L. Girardello, and A. Van Proeyen, Nucl. Phys. B212, 413 (1983).

[9] For a review, see, e.g., H. P. Nilles, Phys. Rep. 110, 1 (1984).

[10] S. Dimopoulos, S. Raby, and F. Wilczek, Phys. Rev. D 24, 1681 (1981)

[11] U. Amaldi, W. de Boer, and H. Fürstenau, Phys. Lett. B 260, 447 (1991).

[12] P. Langacker and M. Luo, Phys. Rev. D 44, 817 (1991).

[13] J. R. Ellis, S. Kelley, and D. V. Nanopoulos, Phys. Lett. B 260, 131 (1991)

[14] S. Heinemeyer, W. Hollik, D. Stockinger, A. M. Weber, and G. Weiglein, J. High Energy Phys. 08 (2006) 052.

[15] L. E. Ibanez and G. G. Ross, Phys. Lett. 110B, 215 (1982).

[16] K. Inoue, A. Kakuto, H. Komatsu, and S. Takeshita, Prog. Theor. Phys. 68, 927 (1982); 70, 330(E) (1983).

[17] J. R. Ellis, J. S. Hagelin, D. V. Nanopoulos, and K. Tamvakis, Phys. Lett. 125B, 275 (1983).

[18] L. Alvarez-Gaume, J. Polchinski, and M. B. Wise, Nucl. Phys. B221, 495 (1983).

[19] G. Velev et al. (Tevatron Electroweak Working Group and for the CDF and D0 Collaborations), arXiv:1107.5255.

[20] CMS Collaboration, Phys. Lett. B 716, 30 (2012).

[21] ATLAS Collaboration, Phys. Lett. B 716, 1 (2012).

[22] T. Aaltonen et al. (CDF and D0 Collaborations), Phys. Rev. Lett. 109, 071804 (2012).

[23] B. W. Lee, C. Quigg, and H. B. Thacker, Phys. Rev. D 16, 1519 (1977).

[24] For a review, see A. Djouadi, Eur. Phys. J. C 59, 389 (2009).

[25] F. D. Steffen, Eur. Phys. J. C 59, 557 (2009).

[26] K.-Y. Choi, J. E. Kim, H. M. Lee, and O. Seto, Phys. Rev. D 77, 123501 (2008).
[27] H. Baer, A. Lessa, S. Rajagopalan, and W. Sreethawong, J. Cosmol. Astropart. Phys. 06 (2011) 031.

[28] H. Baer, A. Lessa, and W. Sreethawong, J. Cosmol. Astropart. Phys. 01 (2012) 036.

[29] K. Rajagopal, M. S. Turner, and F. Wilczek, Nucl. Phys. B358, 447 (1991).

[30] L. Covi, H.-B. Kim, J. E. Kim, and L. Roszkowski, J. High Energy Phys. 05 (2001) 033.

[31] H. Baer, A. D. Box, and H. Summy, J. High Energy Phys. 08 (2009) 080.

[32] D. Curtin, P. Jaiswal, and P. Meade, arXiv:1203.2932.

[33] W. Buchmüller, R. D. Peccei, and T. Yanagida, Annu. Rev. Nucl. Part. Sci. 55, 311 (2005).

[34] I. Affleck and M. Dine, Nucl. Phys. B249, 361 (1985).

[35] M. Dine, L. Randall, and S. D. Thomas, Nucl. Phys. B458, 291 (1996).

[36] CMS Collaboration, Report No. CMS-PAS-SUS-12-028.

[37] P. Bechtle, K. Desch, M. Uhlenbrock, and P. Wienemann, Eur. Phys. J. C 66, 215 (2010).

[38] O. Buchmüller et al., Eur. Phys. J. C 71, 1583 (2011).

[39] A. Arbey, M. Battaglia, A. Djouadi, F. Mahmoudi, and J. Quevillon, Phys. Lett. B 708, 162 (2012).

[40] H. Baer, V. Barger, and A. Mustafayev, J. High Energy Phys. 05 (2012) 091.

[41] K. R. Dienes and C. F. Kolda, arXiv:hep-ph/9712322.

[42] F. Gabbiani, E. Gabrielli, A. Masiero, and L. Silvestrini, Nucl. Phys. B477, 321 (1996).

[43] V.S. Kaplunovsky and J. Louis, Phys. Lett. B 306, 269 (1993).

[44] S. Dimopoulos and H. Georgi, Nucl. Phys. B193, 150 (1981).

[45] Y. Nir and N. Seiberg, Phys. Lett. B 309, 337 (1993).

[46] M. Dine, A. Kagan, and S. Samuel, Phys. Lett. B 243, 250 (1990).

[47] A. G. Cohen, D. B. Kaplan, and A. E. Nelson, Phys. Lett. B 388, 588 (1996).

[48] N. Arkani-Hamed and H. Murayama, Phys. Rev. D 56, R6733 (1997).

[49] H. Murayama and A. Pierce, Phys. Rev. D 65, 055009 (2002).

[50] J. Hisano, D. Kobayashi, T. Kuwahara, and N. Nagata, J. High Energy Phys. 07 (2013) 038. 
[51] S. Weinberg, Phys. Rev. Lett. 48, 1303 (1982).

[52] M. Y. Khlopov and A. D. Linde, Phys. Lett. 138B, 265 (1984).

[53] M. Kawasaki, K. Kohri, T. Moroi, and A. Yotsuyanagi, Phys. Rev. D 78, 065011 (2008).

[54] G. W. Anderson and D. J. Castano, Phys. Rev. D 52, 1693 (1995).

[55] S. Cassel, D. M. Ghilencea, and G. G. Ross, Phys. Lett. B 687, 214 (2010).

[56] R. Kitano and Y. Nomura, Phys. Rev. D 73, 095004 (2006).

[57] R. Kitano and Y. Nomura, Phys. Lett. B 631, 58 (2005).

[58] C. Brust, A. Katz, S. Lawrence, and R. Sundrum, J. High Energy Phys. 03 (2012) 103.

[59] M. Papucci, J. T. Ruderman, and A. Weiler, J. High Energy Phys. 09 (2012) 035.

[60] J. L. Feng, arXiv:1302.6587 [Annu. Rev. Nucl. Part. Sci. (to be published)].

[61] H. Baer, V. Barger, P. Huang, D. Mickelson, A. Mustafayev, and X. Tata, arXiv:1306.2926.

[62] H. Baer, V. Barger, P. Huang, A. Mustafayev, and X. Tata, Phys. Rev. Lett. 109, 161802 (2012).

[63] H. Baer, V. Barger, P. Huang, D. Mickelson, A. Mustafayev, and X. Tata, Phys. Rev. D 87, 035017 (2013).

[64] H. Baer, V. Barger, P. Huang, D. Mickelson, A. Mustafayev, and X. Tata, Phys. Rev. D 87, 115028 (2013).

[65] G. W. Bennett et al. (Muon G-2 Collaboration), Phys. Rev. D 73, 072003 (2006).

[66] M. Davier, A. Hoecker, B. Malaescu, and Z. Zhang, Eur. Phys. J. C 71, 1515 (2011).

[67] T. Moroi, Phys. Rev. D 53, 6565 (1996); 56, 4424(E) (1997).

[68] J. L. Feng and K. T. Matchev, Phys. Rev. Lett. 86, 3480 (2001).

[69] Y. Amhis et al. (Heavy Flavor Averaging Group Collaboration), arXiv:1207.1158.

[70] M. Misiak et al., Phys. Rev. Lett. 98, 022002 (2007).

[71] H. Baer and M. Brhlik, Phys. Rev. D 55, 3201 (1997).

[72] R. Aaij et al. (LHCb Collaboration), Phys. Rev. Lett. 110, 021801 (2013).

[73] G. Buchalla, A. J. Buras, and M. Lautenbacher, Rev. Mod. Phys. 68, 1125 (1996).

[74] K.S. Babu and C.F. Kolda, Phys. Rev. Lett. 84, 228 (2000).

[75] J. K. Mizukoshi, X. Tata, and Y. Wang, Phys. Rev. D 66, 115003 (2002).

[76] D. Eriksson, F. Mahmoudi, and O. Stal, J. High Energy Phys. 11 (2008) 035.

[77] B. W. Lee and S. Weinberg, Phys. Rev. Lett. 39, 165 (1977).

[78] E. A. Baltz, M. Battaglia, M. E. Peskin, and T. Wizansky, Phys. Rev. D 74, 103521 (2006).

[79] H. Goldberg, Phys. Rev. Lett. 50, 1419 (1983); 103, 099905(E) (2009).

[80] J. R. Ellis, J. S. Hagelin, D. V. Nanopoulos, K. A. Olive, and M. Srednicki, Nucl. Phys. B238, 453 (1984).

[81] For a review, see G. Jungman, M. Kamionkowski, and K. Griest, Phys. Rep. 267, 195 (1996).

[82] C. L. Bennett, D. Larson, J.L. Weiland, N. Jarosik, G. Hinshaw, N. Odegard, K. M. Smith, R. S. Hill et al., arXiv: 1212.5225 .
[83] P. A. R. Ade et al. (Planck Collaboration), arXiv:1303.5076.

[84] H. Baer, A. D. Box, and H. Summy, J. High Energy Phys. 10 (2010) 023.

[85] H. Baer and A. D. Box, Eur. Phys. J. C 68, 523 (2010).

[86] LEPSUSYWG, ALEPH, DELPHI, L3, and OPAL experiments, Report No. LEPSUSYWG/04-01.1 [http:// lepsusy.web.cern.ch/lepsusy/Welcome.html]; A. Heister et al. (ALEPH Collaboration), Phys. Lett. B 526, 206 (2002); J. Abdallah et al. (DELPHI Collaboration), Eur. Phys. J. C 31, 421 (2003); P. Achard et al. (L3 Collaboration), Phys. Lett. B 580, 37 (2004); G. Abbiendi et al. (OPAL Collaboration), Eur. Phys. J. C 32, 453 (2004).

[87] B. S. Acharya, G. Kane, and E. Kuflik, arXiv:1006.3272.

[88] T. Moroi and L. Randall, Nucl. Phys. B570, 455 (2000).

[89] G. Gelmini, P. Gondolo, A. Soldatenko, and C. E. Yaguna, Phys. Rev. D 74, 083514 (2006).

[90] R. D. Peccei, Lect. Notes Phys. 741, 3 (2008).

[91] J. E. Kim and G. Carosi, Rev. Mod. Phys. 82, 557 (2010).

[92] L.F. Abbott and P. Sikivie, Phys. Lett. 120B, 133 (1983).

[93] J. Preskill, M. B. Wise, and F. Wilczek, Phys. Lett. 120B, 127 (1983).

[94] M. Dine and W. Fischler, Phys. Lett. 120B, 137 (1983).

[95] H. P. Nilles and S. Raby, Nucl. Phys. B198, 102 (1982).

[96] K. J. Bae, H. Baer, and A. Lessa, J. Cosmol. Astropart. Phys. 04 (2013) 041.

[97] A. Bottino, F. Donato, N. Fornengo, and S. Scopel, Phys. Rev. D 69, 037302 (2004).

[98] O. Adriani et al. (PAMELA Collaboration), Nature (London) 458, 607 (2009).

[99] M. Ackermann et al. (Fermi LAT Collaboration), Phys. Rev. Lett. 108, 011103 (2012).

[100] M. Aguilar et al. (AMS Collaboration), Phys. Rev. Lett. 110, 141102 (2013).

[101] S. Profumo, Central Eur. J. Phys. 10, 1 (2012).

[102] V. Barger, Y. Gao, W. Y. Keung, D. Marfatia, and G. Shaughnessy, Phys. Lett. B 678, 283 (2009).

[103] T. Bringmann, X. Huang, A. Ibarra, S. Vogl, and C. Weniger, J. Cosmol. Astropart. Phys. 07 (2012) 054.

[104] C. Weniger, J. Cosmol. Astropart. Phys. 08 (2012) 007.

[105] T. Cohen, M. Lisanti, T.R. Slatyer, and J. G. Wacker, J. High Energy Phys. 10 (2012) 134.

[106] E. Aprile et al. (XENON100 Collaboration), Phys. Rev. Lett. 109, 181301 (2012).

[107] H. Baer, A. Mustafayev, E.-K. Park, and X. Tata, J. Cosmol. Astropart. Phys. 01 (2007) 017.

[108] J. L. Feng and D. Sanford, J. Cosmol. Astropart. Phys. 05 (2011) 018.

[109] ATLAS Collaboration, Report No. ATLAS-CONF-2013014.

[110] CMS Collaboration, Report No. CMS-PAS-HIG-13-005.

[111] CMS Collaboration, Phys. Lett. B 713, 68 (2012).

[112] ATLAS Collaboration, J. High Energy Phys. 02 (2013) 095.

[113] ATLAS Collaboration, J. High Energy Phys. 06 (2012) 039.

[114] CMS Collaboration, J. High Energy Phys. 07 (2012) 143.

[115] G. Abbiendi et al. (ALEPH and DELPHI and L3 and OPAL and The LEP working group for Higgs boson searches Collaborations), arXiv:1301.6065. 
[116] H. Baer, V. Barger, and A. Mustafayev, Phys. Rev. D 85, 075010 (2012).

[117] S. Heinemeyer, O. Stal, and G. Weiglein, Phys. Lett. B 710, 201 (2012).

[118] P. Bechtle et al., J. High Energy Phys. 06 (2012) 098.

[119] O. Buchmüller et al., Eur. Phys. J. C 72, 2243 (2012).

[120] L. J. Hall, D. Pinner, and J. T. Ruderman, J. High Energy Phys. 04 (2012) 131.

[121] S. F. King, M. Mühlleitner, and R. Nevzorov, Nucl. Phys. B860, 207 (2012).

[122] S. P. Martin, Phys. Rev. D 81, 035004 (2010).

[123] R. Benbrik, M. Gomez Bock, S. Heinemeyer, O. Stal, G. Weiglein, and L. Zeune, Eur. Phys. J. C 72, 2171 (2012).

[124] P. Bechtle, S. Heinemeyer, O. Stal, T. Stefaniak, G. Weiglein, and L. Zeune, Eur. Phys. J. C 73, 2354 (2013).

[125] M. Drees, Phys. Rev. D 86, 115018 (2012).

[126] ATLAS Collaboration, Report No. ATLAS-CONF-2013047.

[127] CMS Collaboration, Report No. arXiv:1303.2985.

[128] ATLAS Collaboration, Report No. ATLAS-CONF-2013054.

[129] ATLAS Collaboration, Report No. ATLAS-CONF-2013007.

[130] CMS Collaboration, arXiv:1305.2390.

[131] CMS Collaboration, J. High Energy Phys. 03 (2013) 037.

[132] CMS Collaboration, Report No. CMS-PAS-SUS-13-007.

[133] CMS Collaboration, Report No. CMS-PAS-SUS-13-008.

[134] H. K. Dreiner, M. Kramer, and J. Tattersall, Europhys. Lett. 99, 61001 (2012).

[135] ATLAS Collaboration, Report No. ATLAS-CONF-2013024.

[136] ATLAS Collaboration, Report No. ATLAS-CONF-2013037.

[137] CMS Collaboration, Report No. CMS-PAS-SUS-13-011.

[138] ATLAS Collaboration, Report No. ATLAS-CONF-2013048.

[139] ATLAS Collaboration, Report No. ATLAS-CONF-2013053.

[140] CMS Collaboration, Report No. CMS-PAS-SUS-13-008.

[141] ATLAS Collaboration, Report No. ATLAS-CONF-2013025.

[142] ATLAS Collaboration, Report No. ATLAS-CONF-2013049.

[143] ATLAS Collaboration, Report No. ATLAS-CONF-2013035.

[144] ATLAS Collaboration, Report No. ATLAS-CONF-2013028.

[145] CMS Collaboration, Report No. CMS-PAS-SUS-12-022.

[146] LEP2 SUSY Working Group, ALEPH, DELPHI, L3, and OPAL experiments, Reports No. LEPSUSYWG/01-03.1 and No. LEPSUSYWG/02-04.1 [http://lepsusy.web.cern.ch/ lepsusy/Welcome.html].

[147] H. Baer, C.-h. Chen, F. Paige, and X. Tata, Phys. Rev. D 50, 4508 (1994).

[148] H. Baer, V. Barger, P. Huang, D. Mickelson, A. Mustafayev, W. Sreethawong, and X. Tata, Phys. Rev. Lett. 110, 151801 (2013).

[149] H. Baer, C.-H. Chen, F. Paige, and X. Tata, Phys. Rev. D 49, 3283 (1994).
[150] http://www-flc.desy.de/ilcphysics/postLHC8-SUSYbenchmarks .php.

[151] A. Buckley, arXiv:1305.4194.

[152] H. Baer, V. Barger, P. Huang, and X. Tata, J. High Energy Phys. 05 (2012) 109.

[153] F. E. Paige, S. D. Protopopescu, H. Baer, and X. Tata, arXiv:hep-ph/0312045.

[154] H. Baer, A. Belyaev, T. Krupovnickas, and X. Tata, J. High Energy Phys. 02 (2004) 007.

[155] H. Baer, T. Krupovnickas, and X. Tata, J. High Energy Phys. 06 (2004) 061.

[156] C. Bartels, M. Berggren, and J. List, Eur. Phys. J. C 72, 2213 (2012).

[157] C. Bartels, O. Kittel, U. Langenfeld, and J. List, arXiv:1202.6324.

[158] H. Baer, V. Barger, and P. Huang, J. High Energy Phys. 11 (2011) 031.

[159] J. A. Aguilar-Saavedra et al. (ECFA/DESY LC Physics Working Group), arXiv:hep-ph/0106315.

[160] K. L. Chan, U. Chattopadhyay, and P. Nath, Phys. Rev. D 58, 096004 (1998).

[161] F. Brummer and W. Buchmuller, J. High Energy Phys. 05 (2012) 006.

[162] H. Baer, V. Barger, A. Lessa, W. Sreethawong, and X. Tata, Phys. Rev. D 85, 055022 (2012).

[163] ATLAS Collaboration, Report No. CERN-LHCC-99-15, especially Figs. 19-82, and Fig 2.5.1 of [159].

[164] T. Abe et al. (ILD Concept Group-Linear Collider Collaboration), arXiv:1006.3396.

[165] H. Aihara, P. Burrows, and M. Oreglia, arXiv:0911.0006.

[166] R. H. K. Kadala, P. G. Mercadante, J. K. Mizukoshi, and X. Tata, Eur. Phys. J. C 56, 511 (2008).

[167] H. Baer, V. Barger, S. Kraml, A. Lessa, W. Sreethawong, and X. Tata, J. High Energy Phys. 03 (2012) 092.

[168] H. Baer, F.E. Paige, S. D. Protopopescu, and X. Tata, arXiv:hep-ph/9305342.

[169] A. Djouadi, J.-L. Kneur, and G. Moultaka, Comput. Phys. Commun. 176, 426 (2007).

[170] W. Porod, Comput. Phys. Commun. 153, 275 (2003).

[171] W. Porod and F. Staub, Comput. Phys. Commun. 183, 2458 (2012).

[172] G. Belanger, F. Boudjema, A. Pukhov, and A. Semenov, Comput. Phys. Commun. 149, 103 (2002).

[173] ATLAS Collaboration, Phys. Rev. Lett. 108, 261804 (2012).

[174] P. Bechtle, M. Berggren, J. List, P. Schade, and O. Stempel, Phys. Rev. D 82, 055016 (2010).

[175] H. Baer, A. Bartl, D. Karatas, W. Majerotto, and X. Tata, Int. J. Mod. Phys. A 04, 4111 (1989).

[176] R. Kallosh and A. D. Linde, J. High Energy Phys. 12 (2004) 004

[177] A. Linde, Y. Mambrini, and K. A. Olive, Phys. Rev. D 85, 066005 (2012).

[178] S. Kachru, R. Kallosh, A. D. Linde, and S. P. Trivedi, Phys. Rev. D 68, 046005 (2003).

[179] L. J. Hall and Y. Nomura, J. High Energy Phys. 01 (2012) 082.

[180] B. S. Acharya, K. Bobkov, G. L. Kane, J. Shao, and P. Kumar, Phys. Rev. D 78, 065038 (2008).

[181] H. Baer, C. Balazs, A. Belyaev, T. Krupovnickas, and X. Tata, J. High Energy Phys. 06 (2003) 054. 
[182] B. C. Allanach, Comput. Phys. Commun. 143, 305 (2002).

[183] Post LHC7 benchmark points are located at http://wwwflc.desy.de/ilcphysics/postLHC7-SUSYbenchmarks.php.

[184] M. Berggren, F. Brmmer, J. List, G. Moortgat-Pick, T. Robens, K. Rolbiecki, and H. Sert, arXiv:1307.3566.
[185] H. Baer, A. Belyaev, T. Krupovnickas, and A. Mustafayev, J. High Energy Phys. 06 (2004) 044.

[186] M. Carena, S. Heinemeyer, O. Stål, C. E. M. Wagner, and G. Weiglein, arXiv:1302.7033.

[187] B. C. Allanach et al., Eur. Phys. J. C 25, 113 (2002). 